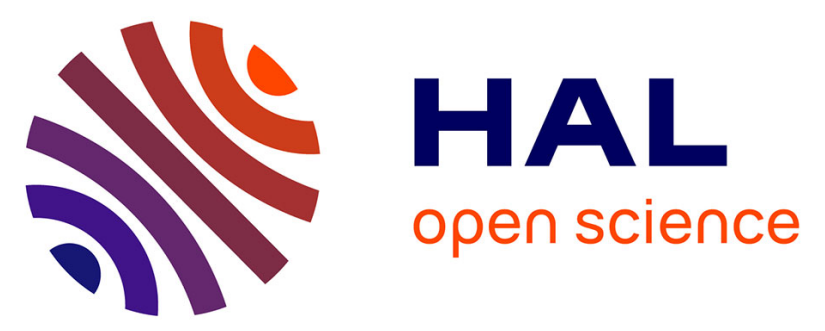

\title{
EXISTENCE AND UNIQUENESS OF GENERALIZED SOLUTIONS TO HYPERBOLIC SYSTEMS WITH LINEAR FLUXES AND STIFF SOURCES
}

Teddy Pichard, Nina Aguillon, Bruno Després, Edwige Godlewski, Michael Ndjinga

\section{To cite this version:}

Teddy Pichard, Nina Aguillon, Bruno Després, Edwige Godlewski, Michael Ndjinga. EXISTENCE AND UNIQUENESS OF GENERALIZED SOLUTIONS TO HYPERBOLIC SYSTEMS WITH LINEAR FLUXES AND STIFF SOURCES. Journal of Hyperbolic Differential Equations, inPress, 18 (3), https://doi.org/10.1142/S021989162150020X. hal-02920155

\section{HAL Id: hal-02920155 \\ https://hal.science/hal-02920155}

Submitted on 24 Aug 2020

HAL is a multi-disciplinary open access archive for the deposit and dissemination of scientific research documents, whether they are published or not. The documents may come from teaching and research institutions in France or abroad, or from public or private research centers.
L'archive ouverte pluridisciplinaire HAL, est destinée au dépôt et à la diffusion de documents scientifiques de niveau recherche, publiés ou non, émanant des établissements d'enseignement et de recherche français ou étrangers, des laboratoires publics ou privés. 
August 24, $2020 \quad 14: 22 \quad$ theory hal

\title{
EXISTENCE AND UNIQUENESS OF GENERALIZED SOLUTIONS TO HYPERBOLIC SYSTEMS WITH LINEAR FLUXES AND STIFF SOURCES
}

\author{
TEDDY PICHARD* \\ Centre de Mathématiques Appliquées, École polytechnique, CNRS UMR7641, \\ Institut Polytechnique de Paris, Route de Saclay, Palaiseau, 91120, France. \\ teddy.pichard@polytechnique.edu \\ NINA AGUILLON \\ Laboratoire Jacques-Louis Lions, Sorbonne-Université, CNRS UMR7598, \\ Université de Paris, 4 place Jussieu, 75005 Paris, France. \\ aguillon@ljll.math.upmc.fr \\ BRUNO DESPRÉS \\ Laboratoire Jacques-Louis Lions, Sorbonne-Université, CNRS UMR7598, \\ Université de Paris, 4 place Jussieu, 75005 Paris, France. \\ despres@ljll.math.upmc.fr \\ EDWIGE GODLEWSKI \\ Laboratoire Jacques-Louis Lions, Sorbonne-Université, CNRS UMR7598, \\ Université de Paris, 4 place Jussieu, 75005 Paris, France. \\ godlewski@ljll.math.upmc.fr \\ MICHAEL NDJINGA \\ CEA Saclay, DEN, 91191, Gif-sur-Yvette, France. \\ michael.ndjinga@cea.fr \\ Received (Day Mth. Year) \\ Revised (Day Mth. Year) \\ Communicated by [editor]
}

\begin{abstract}
Motivated by the modelling of boiling two-phase flows, we study systems of balance laws with a source term defined as a discontinuous function of the unknown. Due to this discontinuous source term, the classical theory of partial differential equations (PDE) is not sufficient here. Restricting to a simpler system with linear fluxes, a notion of generalized solution is developed. An important point in the construction of a solution is that the curve along which the source jumps, which we call the boiling curve, must never be tangent to the characteristics. This leads to exhibit sufficient conditions which ensure the existence and uniqueness of a solution in two different situations: first when the initial data is smooth and such that the boiling curve is either overcharacteristic or
\end{abstract}

${ }^{*}$ corresponding author 
subcharacteristic; then with discontinuous initial data in the case of Riemann problems. A numerical illustration is given in this last case.

Keywords: Balance laws; Stiff sources; Generalized solutions.

\section{Introduction}

The starting point is a system of balance laws of the form

$$
\partial_{t} U+\partial_{x} F(U)=\left\{\begin{array}{l}
S^{-} \text {if } h(U) \leq h^{b}, \\
S^{+} \text {if } h(U)>h^{b} .
\end{array}\right.
$$

For instance, a system of the form (1.1) was obtained as a drift-flux model ([21, $19,20,27,3,26])$ for homogenized boiling two-phase flows. The discontinuity of the source term approximates the boiling phenomenon, which is much faster than the time scale of the flow. In this context, the unknown $U$ and the flux $F(U)$ are given by

$$
U=\left(\alpha \rho_{v}, \rho, \rho u, \rho e\right), \quad F(U)=\left(\alpha \rho_{v} u, \rho u, \rho u^{2}+p, \rho u e+p u\right)
$$

where $\alpha \rho_{v}$ is the partial density of vapor, and $(\rho, \rho u, \rho e)$ are the total density, the total momentum and the total energy of the mixture of vapor and liquid. Here, $p(U)$ is some pressure law, and $h(U)$ is an enthalpy function. The liquid is assumed to boil only when the enthalpy $h(U)$ is above the boiling threshold $h^{b}$. The source is chosen of the form

$$
S^{-}=(0,0,0, \phi), \quad S^{+}=(K \phi, 0,0, \phi),
$$

which corresponds to imposing a source $\phi>0$ of energy everywhere and a creation $K \phi>0$ of vapor only above the boiling threshold $h(U)>h^{b}$. See e.g. [9] for further thermodynamics consideration on the construction of such boiling models.

Most of the results from the "classical theory" require the source term $S(U)$ to be Lipschitz-continuous, and are therefore unavailable here. For instance, in a simple ordinary differential equation (ODE) framework (consider e.g. a $0 \mathrm{D}$ version of (1.1) or see examples in Section 2), the Picard-Lindelöf theorem cannot be applied and neither uniqueness nor existence of a solution to such an ODE is ensured. Even the notion of "classical solution" is unclear and one typically works with the integral notion of Carathodory solutions ([11]). In the literature, several generalizations of the Picard-Lindelöf theorem provide similar results under weaker regularity requirements on the source term, e.g. under directional continuity or bounded variations $([25,5,7,18])$. Other approaches provide results in a more general context, e.g. in Filippov theory $([13,2,8])$, the ODE is replaced by a differential inclusion, and a wider set of solutions is sought. However, these results mainly apply to ODE problems and there are few generalizations to PDE, to the authors' knowledge.

This work focuses on a system with linear fluxes oversimplified compared 
to (1.1), however presenting already interesting features:

$$
\begin{aligned}
& \partial_{t} u-\mu \partial_{x} u=\sigma(u, v), \quad u(0, x)=u_{0}(x), \\
& \partial_{t} v+\nu \partial_{x} v=\varsigma(u, v), \quad v(0, x)=v_{0}(x),
\end{aligned}
$$

with source terms defined by

$$
\sigma(u, v)=\left\{\begin{array}{ll}
a & \text { if } \quad h(u, v) \leq 0, \\
b & \text { if } \quad h(u, v)>0,
\end{array} \quad \varsigma(u, v)=\left\{\begin{array}{lll}
c & \text { if } & h(u, v) \leq 0 \\
d & \text { if } & h(u, v)>0
\end{array}\right.\right.
$$

and where the function $h$ is linear

$$
h(u, v)=h_{1} u+h_{2} v .
$$

For simplicity, the constants are chosen strictly positive

$$
h_{1}>0, \quad h_{2}>0, \quad \mu>0, \quad \nu>0,
$$

though the results below can be generalized. Also, one remarks that the sources $\sigma$ and $\varsigma$ in the two equations are discontinuous at the same $(x, t)$-positions since they both jump when $h(u, v)=0$.

System (1.2) retains the discontinuity of the source with respect to the unknown, but the fluxes and the enthalpy are simplified into linear functions and the system is only composed of two equations. The linearity of $h$ and the reduction of the number of equations is only meant to simplify the mathematical analysis, and the results below could be generalized under weaker but more technical requirements. On the contrary, the hypothesis on the linearity of the fluxes is necessary in the present work. It allows to use the method of characteristics and it prevents the formation of shock and rarefaction waves. Extending the present work with nonlinear fluxes would naturally require to consider such nonlinear waves.

The main results of this paper consist in constructing a solution to (1.2) in three different cases, i.e. subject to various inequalities involving the initial data $u_{0}$ and $v_{0}$ and the source values $a, b, c$ and $d$. The zone of transition $\Gamma$ of the source terms plays an important role in the construction below. This is defined by

$$
\Gamma=\left\{(x, t) \in \mathbb{R} \times \mathbb{R}^{+} \text {s.t. } h(u, v)(x, t)=0\right\} .
$$

In all the cases described in the theorems below, the set $\Gamma$ is a line, and is afterward called the "boiling line". The three sets of conditions required for the well-posedness of (1.2) correspond to imposing that

- for regular initial conditions,

- either $\Gamma$ is an overcharacteristic line, i.e. it remains outside the cones generated by the two characteristics passing at every point $(x, t) \in \Gamma$ (see Fig. 1 top left). A precise definition is given in Section 3.1 and the precise requirements and the result are stated in Theorem 3.3 and the proof is detailed in Section 4; 

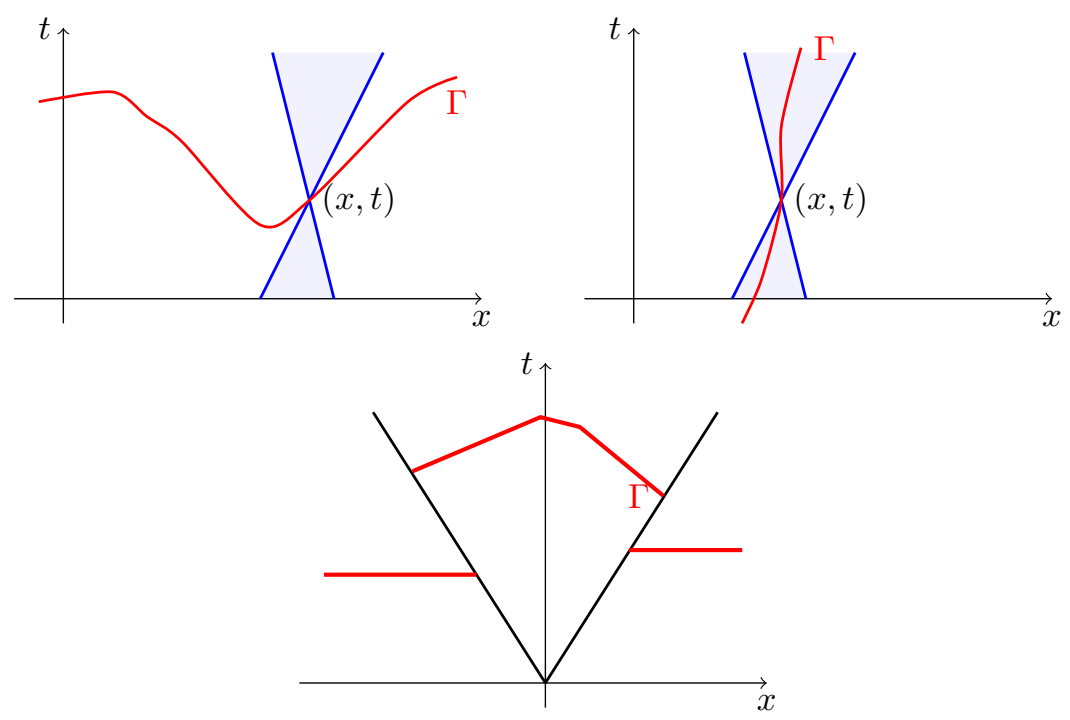

Fig. 1. Examples of boiling lines $\Gamma$ (red) that are overcharacteristic (top left), subcharacteristic (top right) or in the case of Riemann problems (bottom), with the two characteristic curves (blue) passing at a point $(x, t) \in \Gamma$.

- or $\Gamma$ is a subcharacteristic line, i.e. it remains inside the cones generated by the two characteristics passing at every point $(x, t) \in \Gamma$ (see Fig. 1 top right). Similarly the precise definition is given in Section 3.1, the statement in Theorem 3.5 and the proof in Section 5;

- for initial conditions $u_{0}$ and $v_{0}$ that are discontinuous at $x=0$ and constant elsewhere, i.e. in the case of Riemann problems, the set $\Gamma$ is required to be a line which is overcharacteristic in the middle region (see Fig. 1 bottom), the statement of the result is given by Theorem 3.7 and the proof in Section 6 .

We give sufficient conditions for existence and uniqueness of a generalized solution. However, the conditions are not necessary and, as illustrated in a simplified framework in Section 2.1 (the first two cases of this test case), it remains unclear what happens when these conditions are violated.

The paper is organized as follows. In the next section, the main problem and basic ideas are presented, especially the notion of generalized solution is introduced. In Section 3, the notion of non-characteristic boiling line is introduced and the main theorems are presented. The following three sections are devoted to prove these results. Section 7 gathers the concluding comments. 


\section{Generalized solutions}

First, a simplified ODE framework is considered. Then, an extension to PDE's is described.

\subsection{Carathodory solutions for $O D E$}

When considering discontinuous sources in an ODE framework, it is possible to construct solutions when convenient inequalities on the data hold $([23,18,24])$.

Consider the scalar ODE

$$
\frac{d u}{d t}(t)=s(u(t), t), \quad u(0)=u_{0} .
$$

If $s$ is discontinuous at some point $(u, t)$, then the derivative $\frac{d u}{d t}$ is not defined in a classical sense at this point.

Definition 2.1 ([11,23]). Consider $u_{0} \in \mathbb{R}$ and $s \in L^{1}(\mathbb{R} \times] 0, T[)$. A function $u \in W^{1,1}(] 0, T[)$ is a Carathodory solution to (2.1) on $] 0, T[$ if it satisfies

$$
\forall t \in] 0, T\left[, \quad u(t)=u_{0}+\int_{0}^{t} s(u(\tau), \tau) d \tau .\right.
$$

Remark that when $s$ is continuous, then a $C^{1}$ function satisfying (2.1) for all $t \in \mathbb{R}^{+}$also satisfies (2.2). Thus, this definition generalizes the notion of solution to ODE with discontinuous RHS.

Consider now that the source $s=s(u)$ depends only on $u$ and is given by

$$
s(u)= \begin{cases}a & \text { if } u \leq 0, \\ b & \text { otherwise }\end{cases}
$$

The space of parameters $(a, b) \in \mathbb{R}^{2}$ can be decomposed into three subsets. Each of these subsets corresponds to a particular behaviour of the solutions. This can be summarized by the geometrical representation of the solutions on Fig. 2 and by:

- $b \leq 0 \leq a$ and $a \neq b$

The Carathodory solution $u$ is attracted toward $u=0$ (left plot on Fig. 2).

The function

$$
u(t)=\left\{\begin{array}{lll}
u_{0}+a t & \text { if } & u_{0} \leq 0, \\
u_{0}+b t & \text { if } & u_{0} \geq 0,
\end{array}\right.
$$

is the unique Carathodory solution to (2.1-2.3) on $t \in\left[0, T_{\max }[\right.$ with

$$
T_{\max }=\left\{\begin{array}{llll}
\frac{-u_{0}}{a} & \text { if } \quad a \neq 0 \quad \text { and } & u_{0} \leq 0 \\
\frac{-u_{0}}{b} & \text { if } \quad b \neq 0 \quad \text { and } & u_{0} \geq 0 \\
+\infty & \text { otherwise. } & &
\end{array}\right.
$$

This solution (2.4) cannot be prolonged for times $t>T_{\max }$. 
- $a \leq 0 \leq b$

The Carathodory solution $u$ is repulsed away from $u=0$ (right plot on Fig. 2).

If $u_{0} \neq 0$, the function

$$
u(t)=\left\{\begin{array}{lll}
u_{0}+a t & \text { if } & u_{0}<0 \\
u_{0}+b t & \text { if } & u_{0}>0
\end{array}\right.
$$

is the unique Carathodory solution to (2.1-2.3) on $\mathbb{R}^{+}$.

If $u_{0}=0$, both $t \mapsto a t$ and $t \mapsto b t$ are Carathodory solutions to (2.1-2.3) on $t \in \mathbb{R}^{+}$.

- $a b>0$

The Carathodory solution $u$ crosses the discontinuity line $u=0$.

Proposition 2.2. Suppose that $a b>0$. Then, for all $u_{0} \in \mathbb{R}$, there exists a unique Carathodory solution to (2.1-2.3) on $\mathbb{R}^{+}$.

This ensures existence and uniqueness of a Carathodory solution for all $t \in \mathbb{R}^{+}$. This is a straightforward consequence of various more general results, e.g. $[25,5,7,24,17]$. The proof is detailed since its main idea is afterward extended in the PDE framework.

Proof. Suppose that $a>0$ and $b>0$ (similar computations hold if $a<0$ and $b<0$ ). The function (right plot on Fig. 2).

$$
u(t)=\left\{\begin{array}{lll}
u_{0}+b t & \text { if } \quad u_{0}>0, \\
u_{0}+a t & \text { if } \quad u_{0} \leq 0 \quad \text { and } \quad t \leq \frac{-u_{0}}{a}, \\
b\left(t+\frac{u_{0}}{a}\right) & \text { if } \quad u_{0} \leq 0 \quad \text { and } \quad t>\frac{-u_{0}}{a}
\end{array}\right.
$$

is absolutely continuous and satisfies (2.1-2.3) for all $t \in \mathbb{R}^{+}$. Therefore, it is a Carathodory solution to (2.1-2.3) on $\mathbb{R}^{+}$.

Since $s>0$, any Carathodory solution $\tilde{u}$ is strictly increasing. Thus, there exists a unique switch time $t_{s}$ such that $\tilde{u}\left(t_{s}\right)=0$. Thus the source has the form $s(\tilde{u}(t))=\tilde{s}(t)$ with

$$
\tilde{s}(t)=\left\{\begin{array}{lll}
a & \text { if } t \leq t_{s} \\
b & \text { if } t>t_{s} .
\end{array}\right.
$$

Injecting (2.7) in (2.2) leads to a unique $t_{s}=\frac{-u_{0}}{a}$, and thus a unique $\tilde{u}=u$.

The next sections focus on a problem corresponding to a PDE version of the last case.

\subsection{Generalized solutions in a PDE framework}

The PDE framework is formalized in the scalar case, then extended to the case of system (1.2). 

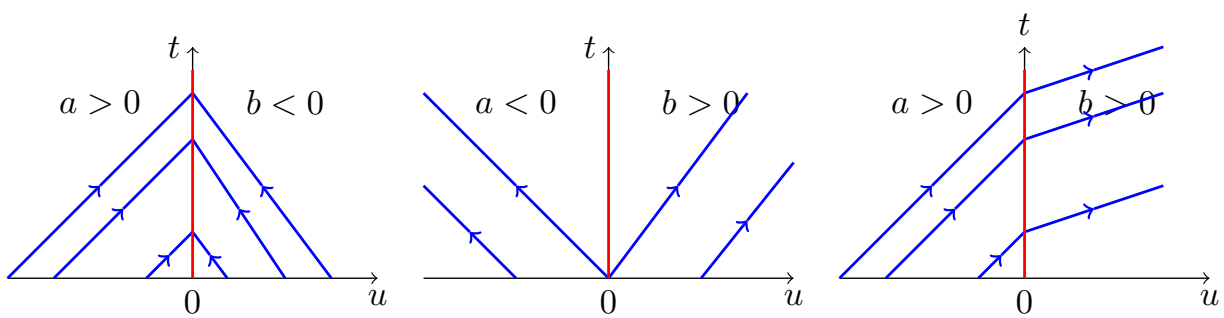

Fig. 2. Representation in phase space $(u, t)$ of the functions $(2.4$; left $),(2.5$; middle) and $(2.6$; right) for different initial conditions $u_{0}$.

\subsubsection{Scalar case}

Consider the scalar equation

$$
\left(\partial_{t} u+\lambda \partial_{x} u\right)(x, t)=s(u(x, t), x, t), \quad u(0, x)=u_{0}(x),
$$

where $\lambda \in \mathbb{R}$ is some given constant. First, the method of characteristics is formalized.

Definition 2.3. The line $C_{\lambda}(x)$, resp. $C_{\lambda}(x, t)$, is defined as the characteristic curve issuing from $(x, 0)$, resp. passing at $(x, t)$. These read

$$
\begin{aligned}
C_{\lambda}(x) & :=\left\{(x+\lambda \tau, \tau), \quad \tau \in \mathbb{R}^{+}\right\}, \\
C_{\lambda}(x, t) & :=\{(x-\lambda \eta, t-\eta), \quad \eta \in]-\infty, t]\} \equiv C_{\lambda}(x-\lambda t),
\end{aligned}
$$

where (2.9a), resp. (2.9b), is written for convenience in the forward, resp. backward, direction.

Following Definition 2.1, a generalized solution is defined as an integral solution along the characteristics.

Definition 2.4. For a function $u \in L_{\text {loc }}^{1}\left(\mathbb{R} \times \mathbb{R}^{+}\right)$, denote

$$
u_{\lambda, x}: \begin{cases}\mathbb{R}^{+} & \rightarrow \mathbb{R} \\ \tau & \mapsto u(x+\lambda \tau, \tau)\end{cases}
$$

Define the set

$$
E_{\lambda}:=\left\{u \in L_{\text {loc }}^{1}\left(\mathbb{R} \times \mathbb{R}^{+}\right) \quad \text { s.t. } \quad \forall x \in \mathbb{R}, \quad u_{\lambda, x} \in W_{\text {loc }}^{1, \infty}\left(\mathbb{R}^{+}\right)\right\} .
$$

Definition 2.5. Consider $u_{0} \in L_{\text {loc }}^{1}(\mathbb{R})$ and $s \in L^{\infty}\left(\mathbb{R} \times \mathbb{R} \times \mathbb{R}^{+}\right)$function of the variables $(u, x, t)$. A function $u \in E_{\lambda}$ is a generalized solution to (2.8) if it satisfies for all $(x, t) \in \mathbb{R} \times \mathbb{R}^{+}$

$$
u(x, t)=u_{0}(x-\lambda t)+\int_{0}^{t} s(u(x-\lambda \eta, t-\eta), x-\lambda \eta, t-\eta) d \eta .
$$


Again, when $s$ is continuous, a function $u$ that is $C^{1}$ and satisfies (2.8) for all $(x, t) \in \mathbb{R} \times \mathbb{R}^{+}$also satisfies (2.11). Thus, this definition generalizes the notion of classical solution. However, solutions to hyperbolic equations are rarely expected to have such a strong regularity, and another notion of generalized solution was introduced in [1], the present one extends it with discontinuous sources for 1D problems.

Remark 2.6. To simplify the analysis in the next sections, two choices about the regularity of the different functions were performed in Definitions 2.4 and 2.5:

- Since all the source terms in our applications are bounded, we have chosen to fix the regularity of $s$ to $L^{\infty}$ in Definition 2.5 and thus we required a $W^{1, \infty}$ regularity in Definition 2.4 along the characteristics. However, weaker integrability of $s$ could have been sufficient to define the integral in (2.11).

On the contrary in the ODE case, for generality (as it is easier to define), the source $s$ was only $L^{1}$ in Definition 2.1, and the regularity of the Carathodory solutions was fixed to $W^{1,1}$.

- The spatial domain was chosen to be the full real line to avoid considering boundary conditions. By extension, the time variable can also go to infinity. However, in most cases we consider below, the integral in (2.11) becomes infinite when $t \rightarrow+\infty$. Then the integrability of the solutions $u \in E_{\lambda}$ can only be local. By extension, as the solution is only locally integrable, there was no point of restricting the initial condition $u_{0}$ with global integrability.

Of course, one could restrict the $(x, t)$-domain into a bounded set (with boundary conditions) and restrict the solution to be in $L^{1}$ such that $u_{\lambda, x}$ is $W^{1, \infty}$. Such a restriction was considered in the ODE in cases as the time domain $] 0, T[$ can be bounded.

1- Case $s$ independent of $u$ : Suppose here that

$$
s(u, x, t)=s(x, t)
$$

with $s \in L^{\infty}\left(\mathbb{R} \times \mathbb{R}^{+}\right)$, i.e. suppose that the source depends only on the variables $(x, t)$ and not on the unknown $u$.

With such a source, the integral on the RHS of (2.11) is well-defined and for all $u_{0} \in L_{l o c}^{1}(\mathbb{R})$, there exists a unique generalized solution to $(2.8)$ on $(x, t) \in \mathbb{R} \times \mathbb{R}^{+}$.

To shorten the writings, a notation for this solution is introduced.

Notation 2.7. Given $\lambda \in \mathbb{R}, u_{0} \in L_{\text {loc }}^{1}(\mathbb{R})$ and $s \in L^{\infty}\left(\mathbb{R} \times \mathbb{R}^{+}\right)$, denote

$$
r_{\lambda}^{u_{0}}(s):\left\{\begin{aligned}
\mathbb{R} \times \mathbb{R}^{+} & \rightarrow \mathbb{R}, \\
(x, t) & \mapsto u_{0}(x-\lambda t)+\int_{0}^{t} s(x-\lambda \eta, t-\eta) d \eta .
\end{aligned}\right.
$$


2- Case $s$ independent of $(\boldsymbol{x}, \boldsymbol{t})$ : Suppose here that $s=s(u)$ with

$$
s(u)= \begin{cases}a & \text { if } u \leq 0 \\ b & \text { otherwise }\end{cases}
$$

where $(a, b) \in \mathbb{R}^{2}$ are given constants.

One easily obtains existence and uniqueness of such a generalized solution.

Corollary 2.8. Suppose that $a b>0$. Then for all $u_{0} \in L_{\text {loc }}^{1}(\mathbb{R})$, there exists $a$ unique generalized solution to (2.8,2.13) on $\mathbb{R} \times \mathbb{R}^{+}$.

Proof. By definition, $u$ is a generalized solution to (2.8) if for all $x \in \mathbb{R}$, the function $t \mapsto u(x+\lambda t, t)$ is a Carathodory solution to (2.1-2.3), where $x$ acts as a parameter. Proposition 2.2 provides the result.

\subsubsection{Case of a system}

Notation 2.9. The dependent variables associated to a system are denoted with a capital letter, e.g. $U=(u, v)$, similarly $\Sigma=(\sigma, \varsigma)$ (also with subscripts or superscripts), while those associated to the sub-equations are denoted with small letters. We will write identically $f(U)=f(u, v)$.

Consider the system of equations in $U=(u, v)$

$$
\begin{aligned}
\left(\partial_{t} u-\mu \partial_{x} u\right)(x, t) & =\sigma(u(x, t), v(x, t), x, t), & u(x, 0)=u_{0}(x), \\
\left(\partial_{t} v+\nu \partial_{x} v\right)(x, t) & =\varsigma(u(x, t), v(x, t), x, t), & v(x, 0)=v_{0}(x) .
\end{aligned}
$$

where $\mu, \nu$ are given constants and $\Sigma$ given sources.

Notation 2.10. Denote

$$
E=E_{-\mu} \times E_{\nu}
$$

Definition 2.11. Consider $U_{0} \in\left(L_{\text {loc }}^{1}(\mathbb{R})\right)^{2}$ and $\Sigma=(\sigma, \varsigma) \in\left(L^{\infty}\left(\mathbb{R}^{2} \times \mathbb{R} \times \mathbb{R}^{+}\right)\right)^{2}$ depending on $(U, x, t)$. Then, a function $U=(u, v) \in E$ is a generalized solution of (2.14) if $U$ satisfies for all $(x, t) \in \mathbb{R} \times \mathbb{R}^{+}$

$$
\begin{array}{r}
u(x, t)=u_{0}(x+\mu t)+\int_{0}^{t} \sigma(u(x+\mu \eta, t-\eta), v(x+\mu \eta, t-\eta), x+\mu \eta, t-\eta) d \eta, \\
v(x, t)=v_{0}(x-\nu t)+\int_{0}^{t} \varsigma(u(x-\nu \eta, t-\eta), v(x-\nu \eta, t-\eta), x-\nu \eta, t-\eta) d \eta .
\end{array}
$$


1- Case $\boldsymbol{\Sigma}$ independent of $\boldsymbol{U}$ : Suppose here that

$$
\forall(U, x, t) \in \mathbb{R}^{2} \times \mathbb{R} \times \mathbb{R}^{+}, \quad \Sigma(U, x, t)=\tilde{\Sigma}(x, t),
$$

where $\tilde{\Sigma} \in\left(L^{\infty}\left(\mathbb{R} \times \mathbb{R}^{+}\right)\right)^{2}$ depends only on $(x, t) \in \mathbb{R} \times \mathbb{R}^{+}$and not on the unknown $U$.

With such a source, the integrals on the RHS of (2.15) are well-defined. Thus, for all $U_{0} \in\left(L_{l o c}^{1}(\mathbb{R})\right)^{2}$, there exists a unique generalized solution $U$ to $(2.8)$ on $(x, t) \in \mathbb{R} \times \mathbb{R}^{+}$.

Notation 2.12. Given $U_{0}=\left(u_{0}, v_{0}\right) \in\left(L_{l o c}^{1}(\mathbb{R})\right)^{2}$ and $\tilde{\Sigma}=(\tilde{\sigma}, \tilde{\varsigma}) \in\left(L^{\infty}(\mathbb{R} \times\right.$ $\left.\left.\mathbb{R}^{+}\right)\right)^{2}$, using the notation 2.7 , denote

$$
R(\tilde{\Sigma})=\left(r_{-\mu}^{u_{0}}(\tilde{\sigma}), r_{\nu}^{v_{0}}(\tilde{\varsigma})\right) .
$$

2- Case $\boldsymbol{\Sigma}$ independent of $(\boldsymbol{x}, \boldsymbol{t})$ : Coming back to (1.2) with a source $\Sigma=$ $(\sigma, \varsigma)$ of the form (1.2c), i.e. depending only on the unknown $U$, then the integral equation (2.15) rewrites

$$
U=R(\Sigma(U)) .
$$

Notation 2.13. Emphasizing the dependence on $U$, note $\Gamma(U)$ the set $\Gamma$ defined in (1.3).

In the present work, only cases where $\Gamma(U)$ is a line are considered.

Notation 2.14. Suppose that $U=(u, v) \in E$ is such that $\Gamma(U)$ is a line. The $(x, t)$-space is decomposed into two zones separated by $\Gamma(U)$

$Z^{+}(U):=\left\{(x, t) \in \mathbb{R} \times \mathbb{R}^{+}\right.$s.t. $\left.h(u, v)(x, t)>0\right\}, \quad Z^{-}(U):=\mathbb{R} \times \mathbb{R}^{+} \backslash Z^{+}(U)$.

In such a case, the boiling line satisfies $\Gamma(U)=\overline{Z^{+}(U)} \cap Z^{-}(U)$.

When $\Sigma$ depends $U$, the two equations of (2.15) are coupled. Indeed, the evaluation of both integrals in the right-hand side require the value of both $U=(u, v)$ in all the so-called cone of dependence ([6] (in pale blue on Fig. 3) delimited by $C_{-\mu}(x, t)$ and $C_{\nu}(x, t)$, and no straightforward application of Corollary 2.8 provides existence and uniqueness of a generalized solution. This problem requires further investigations which are described below.

\subsection{Construction of a generalized solution}

First, the method is presented as a prediction-correction technique, then it is illustrated on a numerical example.

\subsubsection{Prediction-correction method}

A generalized solution $U \in E$ of (1.2) is constructed based on a prediction $\tilde{U}$ which is corrected into $\bar{U}$, then sufficient conditions are exhibited which ensure that $\bar{U}$ is solution. 


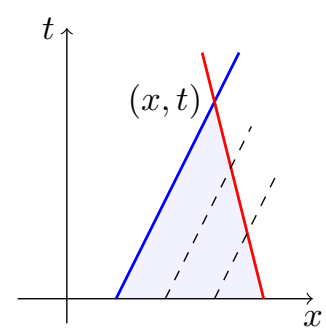

Fig. 3. Representation of a cone of dependence (pale blue) at a point $(x, t)$ delimited by $C_{-\mu}(x, t)$ (red) and $C_{\nu}(x, t)$ (dark blue); and $C_{\nu}(\check{x}, \breve{t})$ (dashed) for different $(\check{x}, \check{t}) \in C_{-\mu}(x, t)$.

Step 1: Define the function $\tilde{\Sigma}=(\tilde{\sigma}, \tilde{\varsigma}) \in\left(L^{\infty}\left(\mathbb{R} \times \mathbb{R}^{+}\right)\right)^{2}$ by

$$
\begin{aligned}
& \tilde{\sigma}(x, t)=\sigma\left(U_{0}\right)(x+\mu t)=\left\{\begin{array}{lll}
a & \text { if } & h\left(U_{0}\right)(x+\mu t) \leq 0, \\
b & \text { if } & h\left(U_{0}\right)(x+\mu t)>0,
\end{array}\right. \\
& \tilde{\varsigma}(x, t)=\varsigma\left(U_{0}\right)(x-\nu t)=\left\{\begin{array}{lll}
c & \text { if } & h\left(U_{0}\right)(x-\nu t) \leq 0, \\
d & \text { if } & h\left(U_{0}\right)(x-\nu t)>0 .
\end{array}\right.
\end{aligned}
$$

The source $\tilde{\Sigma}$ can be interpreted as the source $\Sigma$ at small time, before the switch indicator $h(U)$ has changed sign. Indeed, the source $\tilde{\sigma}$, resp. $\tilde{\varsigma}$, has in $(x, t)$ the value of $\sigma$, resp. $\varsigma$, at the foot of the characteristics $C_{-\mu}(x, t)$, resp. $C_{\nu}(x, t)$.

Step 2: Solve

$$
\begin{aligned}
\partial_{t} \tilde{u}-\mu \partial_{x} \tilde{u} & =\tilde{\sigma}(x, t), \quad \tilde{u}(x, 0)=u_{0}(x), \\
\partial_{t} \tilde{v}+\nu \partial_{x} \tilde{v}=\tilde{\varsigma}(x, t), & \tilde{v}(x, 0)=v_{0}(x),
\end{aligned}
$$

or equivalently compute

$$
\tilde{U}=R(\tilde{\Sigma}),
$$

where $\tilde{\Sigma}=(\tilde{\sigma}, \tilde{\varsigma})$ is independent of the unknown $\tilde{U}=(\tilde{u}, \tilde{v})$. Similarly, $\tilde{U}$ is a prediction of the generalized solution at small times. Indeed, consider that $(x, t)$ is such that $h(U)$ has not changed sign along $C_{-\mu}(x, t)$ and $C_{\nu}(x, t)$, then $\tilde{U}$ satisfies $(2.15)$ at $(x, t)$ and is a generalized solution.

Step 3: Solve

$$
\begin{aligned}
\partial_{t} \bar{u}-\mu \partial_{x} \bar{u} & =\sigma(\tilde{U}(x, t)), & & \bar{u}(x, 0)=u_{0}(x), \\
\partial_{t} \bar{v}+\nu \partial_{x} \bar{v} & =\varsigma(\tilde{U}(x, t)), & & \bar{v}(x, 0)=v_{0}(x) .
\end{aligned}
$$

or equivalently compute

$$
\bar{U}=R \circ \Sigma(\tilde{U}) .
$$

This is a new decoupled system over $\bar{U}=(\bar{u}, \bar{v})$ where the source is computed using $\tilde{U}=(\tilde{u}, \tilde{v})$. Here, $\bar{U}$ corresponds to a correction of (2.18) at larger times. 
There remains to check whether $\bar{U}$ is a generalized solution to (1.2):

Step 4: Check that

$$
\operatorname{sign}(h(\tilde{U}))=\operatorname{sign}(h(\bar{U})) .
$$

By definition, this implies that $Z^{ \pm}(\tilde{U})=Z^{ \pm}(\bar{U})$, and especially this leads to

$$
\Sigma(\tilde{U})=\Sigma(\bar{U}) .
$$

Re-injecting it in (2.19) reads the original system (1.2) and thus $\bar{U}$ is a generalized solution.

In fact, this last step holds only under conditions over the data. Some sufficient conditions are provided in Section 3.

The uniqueness of this generalized solution is also studied a posteriori, once $\bar{U}$ is constructed.

Remark 2.15. An alternative to this approach based on a fixed point method is proposed in [6] with smooth source terms $\Sigma=(\sigma, \varsigma)$. It consists in solving iteratively

$$
\begin{array}{rlrl}
\partial_{t} u^{i+1}-\mu \partial_{x} u^{i+1} & =\sigma\left(u^{i}, v^{i}\right), & & u^{i+1}(0, x)=u_{0}(x), \\
\partial_{t} v^{i+1}+\nu \partial_{x} v^{i+1}=\varsigma\left(u^{i}, v^{i}\right), & v^{i+1}(0, x) & =v_{0}(x),
\end{array}
$$

or equivalently computing $U^{i+1}=R \circ \Sigma\left(U^{i}\right)$, until convergence (if convergence occurs). The present method consists in providing $U^{0}=\tilde{U}$ (in Step 1) in this fixed point such that it converges at the first iteration, i.e. $U^{1}=U^{2}=\ldots=U^{\infty}$.

\subsubsection{Example with numerical values}

Due to the technicality of the computations in the proofs below, the steps of the last subsection are first illustrated on a numerical example. The data are chosen to satisfy the conditions described in the case of Section 3.2 below, i.e. of Theorem 3.3.

Consider the Cauchy problem (1.2) with

$$
\begin{gathered}
h_{1}=h_{2}=\mu=\nu=1, \quad u_{0}(x)=-10, \quad v_{0}(x)=x, \\
a=10, \quad b=20, \quad c=30, \quad d=40,
\end{gathered}
$$

with these data, (1.2) reads

$$
\begin{aligned}
\partial_{t} u-\partial_{x} u=\left\{\begin{array}{rl}
10 & \text { if } u+v \leq 0, \\
20 & \text { if } u+v>0,
\end{array} \quad \partial_{t} v+\partial_{x} v\right. & = \begin{cases}30 & \text { if } u+v \leq 0, \\
40 & \text { if } u+v>0,\end{cases} \\
u_{0}(x) & =-10, \quad v_{0}(x)=x .
\end{aligned}
$$

Step 1: Define for all $(x, t) \in \mathbb{R} \times \mathbb{R}^{+}$

$$
\tilde{\sigma}(x, t)=\left\{\begin{array}{ll}
10 & \text { if }-10+x+t \leq 0, \\
20 & \text { if }-10+x+t>0,
\end{array} \quad \tilde{\varsigma}(x, t)= \begin{cases}30 & \text { if }-10+x-t \leq 0, \\
40 & \text { if }-10+x-t>0,\end{cases}\right.
$$


At initial time, $h\left(U_{0}\right)(x)=0$ only for $x=10$. Thus, $\tilde{\sigma}$ switches value along $C_{-1}(10)$ and $\tilde{\varsigma}$ along $C_{1}(10)$ (see Fig. 4).

Step 2: Integrating these sources along the characteristics provides

$$
\begin{aligned}
\forall(x, t) \in \mathbb{R} \times \mathbb{R}^{+}, \quad \tilde{u}(x, t) & =\left\{\begin{array}{lll}
-10+10 t & \text { if } & -10+x+t \leq 0, \\
-10+20 t & \text { if } & -10+x+t>0,
\end{array}\right. \\
\tilde{v}(x, t) & =\left\{\begin{array}{lll}
x-t+30 t & \text { if } & -10+x-t \leq 0, \\
x-t+40 t & \text { if } & -10+x-t>0 .
\end{array}\right.
\end{aligned}
$$

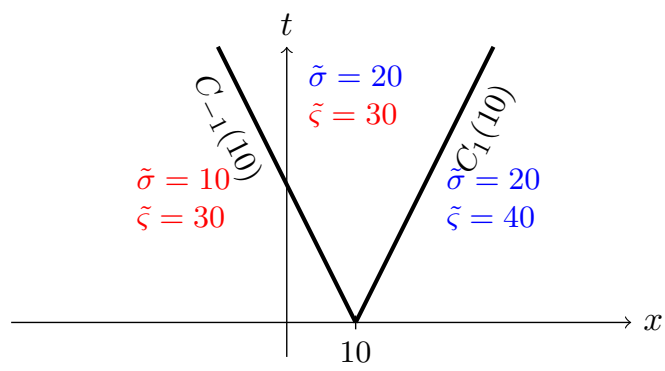

Fig. 4. Representation of the three zones where the source $\tilde{\Sigma}$ has different constant values.

Step 3: Computing $h(\tilde{U})$ yields

$$
\forall(x, t) \in \mathbb{R} \times \mathbb{R}^{+}, \quad h(\tilde{u}, \tilde{v})(x, t)=\left\{\begin{array}{l}
-10+x+39 t \text { if } \quad x \leq 10-t \\
-10+x+49 t \text { if } 10-t<x \leq 10+t, \\
-10+x+59 t \text { otherwise }
\end{array}\right.
$$

Computing where $h(\tilde{U}(x, t))=0$ in $\mathbb{R} \times \mathbb{R}^{+}$provides the line $\Gamma(\tilde{U})$

$$
\left.\left.\Gamma(\tilde{U})=\left\{\left(x, \frac{10-x}{39}\right), \quad x \in\right]-\infty, 10\right]\right\} .
$$

Then $h(\tilde{U})$ is positive above this line (for all $t>T(x)=\frac{10-x}{39}$ and also for all $x>10)$ and negative under. The sign of $h(\tilde{U})$ provides the value $\Sigma(\tilde{U})$, and integrating along the characteristics gives (see Fig. 5)

$$
\begin{aligned}
& \bar{u}(x, t)=\left\{\begin{array}{lll}
-10+10 t \quad \text { if } x \leq 10-39 t & (I), \\
-10+20 t \quad \text { if } x>10-t & (I I), \\
-10+10 t_{s}+20\left(t-t_{s}\right) & \text { otherwise }(I I I),
\end{array} \quad t_{s}=\frac{-10+x+t}{40},\right. \\
& \bar{v}(x, t)= \begin{cases}(x-t)+30 t \quad \text { if } x \leq 10-39 t & (I V), \\
(x-t)+40 t \quad \text { if } x>10+t & (V), \\
(x-t)+30 \tau_{s}+40\left(t-\tau_{s}\right) \text { otherwise }(V I), & \tau_{s}=\frac{-10+x+t}{38} .\end{cases}
\end{aligned}
$$


Step 4: Computing $h(\bar{u}, \bar{v})$ reads for all $(x, t) \in \mathbb{R} \times \mathbb{R}^{+}$

$$
h(\bar{u}, \bar{v})(x, t)= \begin{cases}-10+x+39 t \quad \text { if } x \leq 10-39 t & (I) \cap(I V), \\ -10+x+59 t \quad \text { if } x>10+t & (I I) \cap(V), \\ -10+x+59 t-10 \tau_{s} \text { if } 10+t \geq x>10-t(I I) \cap(V I), \\ -10+x+59 t-10\left(t_{s}+\tau_{s}\right) \quad \text { otherwise } & (I I I) \cap(V I) .\end{cases}
$$

One verifies that $h(\bar{U})$ is negative for $x<10-39 t$, i.e. below $\Gamma(\tilde{U})$ and is positive for $x>\min (10-39 t, 0)$, i.e. above and on the right of $\Gamma(\tilde{U})$. Thus $\bar{U}=(\bar{u}, \bar{v})$ is a generalized solution.
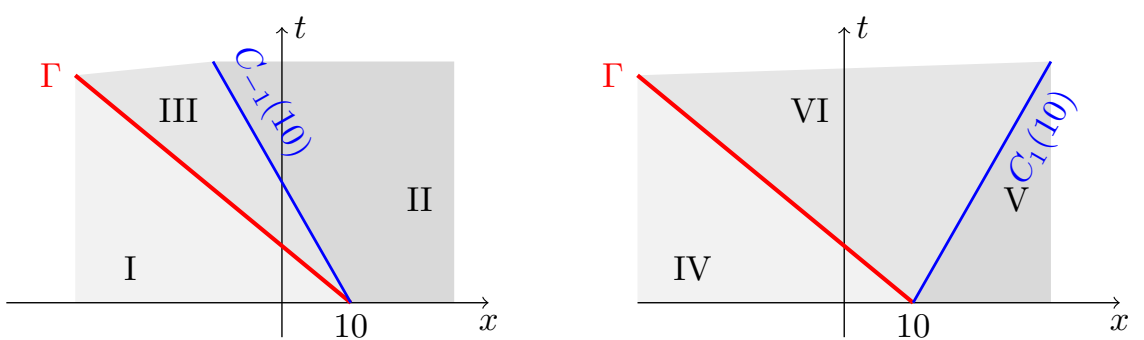

Fig. 5. Zones (I), (II) or (III) (resp. (IV), (V) or (VI)) in which $\bar{u}$ (left), resp. $\bar{v}$ (right), takes the value given in (2.23).

\section{Main results}

First, the notion of non-characteristic curve is presented. Then, conditions are exhibited to ensure that $\Gamma(\tilde{U})$ is non-characteristic, then we obtain existence and uniqueness of a generalized solution.

\subsection{Non-characteristic curves}

The expression non-characteristic is understood here, for a curve $\Gamma$, as the curve being non-tangent to $C_{-\mu}(x, t)$ and to $C_{\nu}(x, t)$ at all point $(x, t) \in \Gamma$. Therefore, the slopes of such tangents to $\Gamma$ are compared to the velocities $-\mu$ and $\nu$.

Two notions of non-characteristic curves are used. The following definitions are adapted from the literature (see e.g. $[12,14,28]$ ) to the present problem.

Definition 3.1 (Overcharacteristic curve). The curve $G=\{(x, T(x)), x \in \mathbb{R}\}$ with $T \in W^{1, \infty}(\mathbb{R})$ is overcharacteristic if

$$
\frac{1}{-\mu}<\inf T^{\prime}, \quad \text { and } \quad \sup T^{\prime}<\frac{1}{\nu} .
$$

This imposes that $G$ does not enter in the cone defined by the characteristics at $(x, t)$ (see Fig. 6, left). 
Imposing that $\Gamma(U)$ is overcharacteristic corresponds to imposing that the boiling front propagates faster than the flow, i.e. $X^{\prime}<-\mu$ or $X^{\prime}>\nu$.

Definition 3.2 (Subcharacteristic curve). The curve $G=\left\{(X(t), t), t \in \mathbb{R}^{+}\right\}$ with $X \in W^{1, \infty}\left(\mathbb{R}^{+}\right)$is subcharacteristic if

$$
-\mu<\inf X^{\prime}, \quad \text { and } \quad \sup X^{\prime}<\nu .
$$

This imposes that the curve $G$ stays inside the cone defined by the characteristics at $(x, t)$ (see Fig. 6, right).

Imposing that $\Gamma(U)$ is subcharacteristic corresponds to imposing that the boiling front propagates with a speed between $-\mu$ and $\nu$.
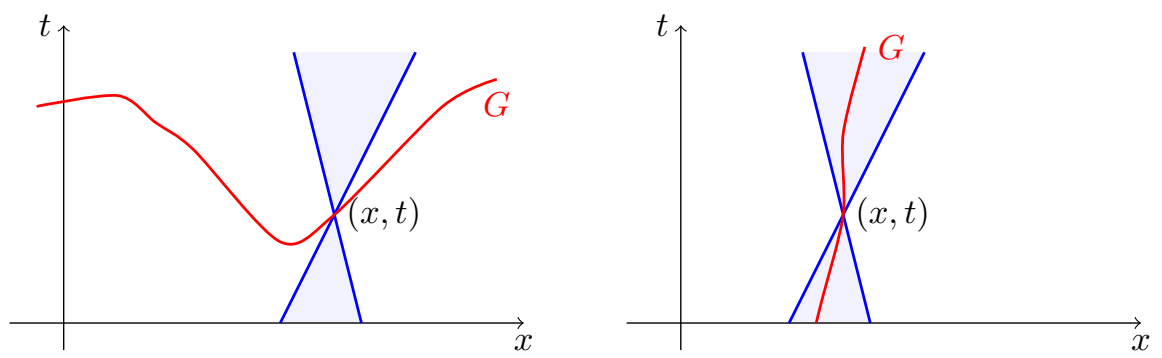

Fig. 6. Examples of an overcharacteristic (3.1; left) and subcharacteristic (3.2; right) curve $G$ in red, and $C_{-\mu}(x, t)$ and $C_{\nu}(x, t)$ passing at $(x, t) \in G$ in blue.

The requirements in the next paragraphs are obtained (after computation) by imposing that $\Gamma(\tilde{U})$ is non-characteristic, where $\tilde{U}$ is the small time solution given in Section (2.3.1). The cases where $\Gamma(\tilde{U})$ is tangent to a characteristic are rejected as it might affect the existence or the uniqueness of the solution for similar reasons as the case $a b \leq 0$ was rejected in the ODE framework of Section 2.1. Indeed, assuming e.g. that $\Gamma(\tilde{U})$ is tangent to $C_{-\mu}(x, t)$ (on a non-empty time interval), then fixing the value of $\Sigma$ in $(x, t)$ to $(a, c)$ or to $(b, d)$ could provide two different $U$ satisfying (2.15).

Theorems 3.3, 3.5 and 3.7 below could be proved using e.g. [5,8]. These theorems are presented in a simplified form for the sake of readability, but more general versions of these well-posedness results are given in Lemmas 4.2, 5.2 and 6.1 which do not fit within the scope of the above cited literature. Furthermore, the present approach provides some additional notions about the solution and its boiling line $\Gamma(U)$.

\subsection{Overcharacteristic case}

Under some specified requirements, (1.2) has a unique generalized solution. 
Theorem 3.3. Suppose that $a \leq b$ and $c \leq d$. Then, for all $\left(u_{0}, v_{0}\right) \in\left(W^{1, \infty}(\mathbb{R})\right)^{2}$ satisfying

$$
\inf u_{0}^{\prime}>-\frac{h(a, c)}{h_{1}(\mu+\nu)}, \quad \sup v_{0}^{\prime}<\frac{h(a, c)}{h_{2}(\mu+\nu)},
$$

there exists a unique generalized solution to (1.2).

This corresponds to the case when the sources in the two equations have higher values when $h(u, v)>0$ than when $h(u, v)<0$. Especially this holds for the application (1.1) we have in mind.

Remark 3.4. In Section 4, the boiling line $\Gamma(U)$ associated to this unique solution $U$ is proved to be globally overcharacteristic under condition (3.3).

In practice, the solution in this case is $\left(W_{l o c}^{1, \infty}\left(\mathbb{R} \times \mathbb{R}^{+}\right)\right)^{2}$ which is a stronger regularity than the requirement to be in $E$.

\subsection{Subcharacteristic case}

Under some specified requirements, (1.2) has a unique generalized solution.

Theorem 3.5. Suppose that $b \leq a$ and $d \leq c$. Then, for all $\left(u_{0}, v_{0}\right) \in\left(W^{1, \infty}(\mathbb{R})\right)^{2}$ satisfying

$$
\inf u_{0}^{\prime}>\frac{-h(b, d)}{h_{1}(\mu+\nu)}, \quad \inf v_{0}^{\prime}>\frac{h(a, c)}{h_{2}(\mu+\nu)},
$$

there exists a unique generalized solution to (1.2).

This corresponds to the case when the sources in the two equations have lower values when $h(u, v)>0$ than when $h(u, v)<0$.

Remark 3.6. In Section 5 , the boiling line $\Gamma(U)$ associated to this unique solution $U$ is proved to be subcharacteristic under condition (3.4). This happens e.g. for a steady solution since the boiling front is stationary with a corresponding boiling curve of the form $\Gamma=\{(X, t), t \in \mathbb{R}\}$ with constant $X$.

In practice, the solution in this case is also $\left(W_{\text {loc }}^{1, \infty}\left(\mathbb{R} \times \mathbb{R}^{+}\right)\right)^{2}$.

\subsection{Riemann problems}

Riemann problems are often studied as they offer a simple setup involving discontinuities in the solution. Furthermore, approximating their solutions leads to the construction of the well-known Godunov-type schemes. Consider initial conditions of the form

$$
u(0, x)=u_{0}(x)=\left\{\begin{array}{ll}
u_{L}^{0} & \text { if } x \leq 0, \\
u_{R}^{0} & \text { otherwise, }
\end{array} \quad v(0, x)=v_{0}(x)= \begin{cases}v_{L}^{0} & \text { if } x \leq 0, \\
v_{R}^{0} & \text { otherwise }\end{cases}\right.
$$


Again, we specify some requirements ensuring existence and uniqueness of a generalized solution.

Theorem 3.7. Suppose that

$$
a<b, \quad c<d \quad \text { and } h(a, c)>0 .
$$

Then, for all $\left(U_{L}^{0}, U_{R}^{0}\right) \in \mathbb{R}^{4}$, there exists a unique generalized solution to (1.2,3.5).

Since a generalized solution $U=(u, v)$ is sought in $E$, then $u$ is Lipschitz continuous along $C_{-\mu}(x, t)$ and $v$ along $C_{\nu}(x, t)$ for all $(x, t) \in \mathbb{R} \times \mathbb{R}^{+}$. However, as for a Riemann problem without source term, $u$ jumps across $C_{-\mu}(0)$ and $v$ across $C_{\nu}(0)$. Thus the solution is constructed differently in the three subsets of $\mathbb{R} \times \mathbb{R}^{+}$ separated by $C_{-\mu}(0)$ and $C_{\nu}(0)$ (see Fig. 7 ).

$$
\begin{aligned}
& D_{L}=\left\{(x, t) \in \mathbb{R} \times \mathbb{R}^{+} \text {s.t. } x \leq-\mu t\right\}, \\
& D_{R}=\left\{(x, t) \in \mathbb{R} \times \mathbb{R}^{+} \text {s.t. } \nu t<x\right\}, \\
& D^{*}=\left\{(x, t) \in \mathbb{R} \times \mathbb{R}^{+} \text {s.t. }-\mu t<x \leq \nu t\right\} .
\end{aligned}
$$

In Section 6, the generalized solution is constructed in the following way:

In $D_{L}$ and $D_{R}$ : The generalized solution $U=(u, v)$ depends only on time

$$
\forall(x, t) \in D_{L}, \quad U(x, t)=U_{L}(t), \quad \forall(x, t) \in D_{R}, \quad U(x, t)=U_{R}(t) .
$$

Furthermore, $U_{L}$, and $U_{R}$ are continuous piecewise affine functions of $t$. Thus, the restrictions of the boiling line in $D_{L}$ and $D_{R}$ are just horizontal (see Fig. 7)

$$
\begin{array}{ll}
\Gamma_{L}:=\left\{\left(y, \tau_{L}\right),\right. & \left.\left.y \in]-\infty, y_{L}\right]\right\} \subset D_{L}, \\
\Gamma_{R}:=\left\{\left(y, \tau_{R}\right),\right. & y \in\left[y_{R},+\infty[\} \subset D_{R},\right.
\end{array}
$$

where $\tau_{L}, \tau_{R}, y_{L}$ and $y_{R}$ are constants to determine. By definition, such lines $\Gamma_{L}$ and $\Gamma_{R}$ are overcharacteristic.

In $D^{*}$ : the solution $U=(u, v)$ is also continuous piecewise affine and the source jumps along a boiling line $\Gamma^{*}$ of the form

$$
\Gamma^{*}:=\left\{(y, T(y)), \quad y \in\left[y_{L}^{*}, y_{R}^{*}\right]\right\} \subset D^{*},
$$

with a function $T$ continuous piecewise affine over $\left[y_{L}^{*}, y_{R}^{*}\right]$ (to determine). In practice, the function $T$ is composed of at most three pieces such that $\Gamma^{*}$ is overcharacteristic (see Fig. 7). The restriction on the source term leads to reject all cases where $\Gamma^{*}$ is subcharacteristic.

\section{Remark 3.8.}

- The condition $h(a, c)>0$ (or (6.1a) below) is imposed to ensure that the jump of the source term $(\sigma, \varsigma)$ does not impact the strict monotonicity of $h(u, v)$ along the characteristics inside $D_{L}, D^{*}$ and $D_{R}$ (in the spirit of Proposition 2.2). 


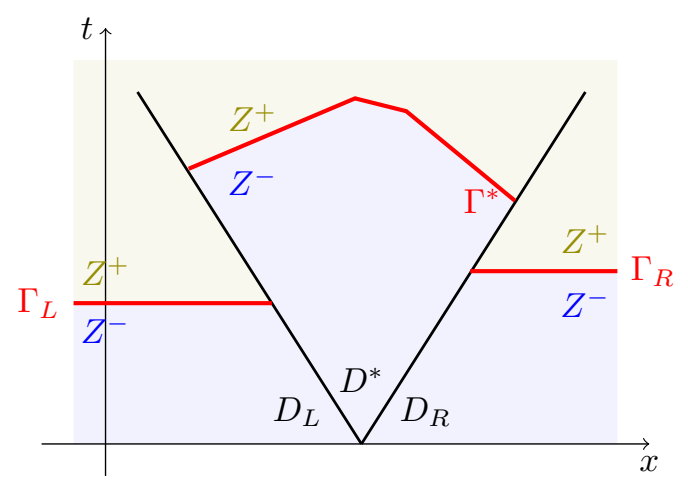

Fig. 7. Example of a boiling curve (in red) in the case of a Riemann problem in Theorem 6.1.

- The conditions $a<b$ and $c<d$ (or (6.1b) and (6.1c) below) are imposed to ensure that the boiling curve $\Gamma^{*}$ in $D^{*}$ is overcharacteristic.

\section{Solution in the regular overcharacteristic case (Theorem 3.3)}

The proof of Theorem 3.3 is based on Lemma 4.2 described in a first subsection. This lemma is proved in the remaining subsections using the technique of Section 2.3.1.

\subsection{Notations and precise statement}

In order to shorten the writings, the following notations are used.

Notation 4.1. We set

$$
\begin{aligned}
& \sigma_{0}(x):=\sigma\left(U_{0}\right)(x), \quad \varsigma_{0}(x):=\varsigma\left(U_{0}\right)(x), \\
& \Theta(y, z):=\frac{-\left[h_{1} u_{0}^{\prime}(y)+h_{2} v_{0}^{\prime}(z)\right]}{h_{1}\left(\mu u_{0}^{\prime}+\sigma_{0}\right)(y)+h_{2}\left(-\nu v_{0}^{\prime}+\varsigma_{0}\right)(z)}, \\
& m:=\inf _{(y, z) \in \mathbb{R}^{2}} \Theta(y, z), \quad M:=\sup _{(y, z) \in \mathbb{R}^{2}} \Theta(y, z) \text {, } \\
& \theta:=\frac{-(\mu+\nu) m}{1-\nu m}, \quad \vartheta \quad:=\frac{(\mu+\nu) M}{1+\mu M} .
\end{aligned}
$$

Lemma 4.2. Suppose that $U_{0} \in\left(W^{1, \infty}(\mathbb{R})\right)^{2}$ is such that

$$
\begin{array}{ccc}
-\frac{1}{\mu}<m, & M<\frac{1}{\nu}, & (4.2 \mathrm{a}) \\
h_{1}(\mu+\nu) \sup _{x}\left(-u_{0}^{\prime}(x)\right)<h_{1} \min (a, b, b+(a-b) \theta)+h_{2} \min (c, d), & (4.2 \mathrm{~b}) \\
h_{2}(\mu+\nu) \sup _{x}\left(v_{0}^{\prime}(x)\right)<h_{1} \min (a, b) & +h_{2} \min (c, d, d+(c-d) \vartheta) .
\end{array}
$$


Then, there exists a unique generalized solution to (1.2).

The method of Section 2.3.2 is followed to prove Lemma 4.2 in the next subsections: first, a function $\bar{U} \in E$ is constructed; second, it is proved to be a generalized solution to (1.2) under the conditions (4.2); third, it is proved to be the only generalized solution.

Remark 4.3. The coefficients $m$ and $M$ are lower and upper bounds of the derivative $T^{\prime}$ of the function $T$ involved in the boiling line $\Gamma(\tilde{U})$, where $\tilde{U}$ is defined in (2.18).

Assuming Lemma 4.2 holds, then Theorem 3.3 is proved:

Proof of Theorem 3.3. First, let us verify that $m$ and $M$ are well-defined under condition (3.3). Write

$\operatorname{Den}(y, z):=h_{1}\left(\mu u_{0}^{\prime}+\sigma_{0}\right)(y)+h_{2}\left(-\nu v_{0}^{\prime}+\varsigma_{0}\right)(z), \quad N u m(y, z):=-\left(h_{1} u_{0}^{\prime}(y)+h_{2} v_{0}^{\prime}(z)\right)$

such that $\Theta=\frac{N u m}{D e n}$. Injecting (3.3) in $\operatorname{Den}(y, z)$ yields

$$
\inf _{(y, z) \in \mathbb{R}^{2}} \operatorname{Den}(y, z)>-h(a, c)+\inf _{(y, z) \in \mathbb{R}^{2}} h_{1} \sigma_{0}(y)+h_{2} \varsigma_{0}(z) \geq 0 .
$$

Now, fixing $\sigma_{0}=a$ and $\varsigma_{0}=c$ in $\Theta$, one observes that the function

$$
(X, Y) \mapsto \frac{-\left(h_{1} X+h_{2} Y\right)}{h_{1}(\mu X+a)+h_{2}(-\nu Y+c)},
$$

is strictly monotonic w.r.t. $X \in]-\frac{h(a, c)}{h_{1}(\mu+\nu)},+\infty[$ and to $Y \in]-\infty, \frac{h(a, c)}{h_{2}(\mu+\nu)}[$.

Thus, the extreme values of $\Theta$ can be found at the extreme values $\sup u_{0}^{\prime}, \sup v_{0}^{\prime}$, $\inf u_{0}^{\prime}$ and $\inf v_{0}^{\prime}$. Computing $\Theta$ with these values of $u_{0}^{\prime}$ and $v_{0}^{\prime}$, one finds that (4.2a) holds. Similar computations hold with the other values of $\sigma_{0}$ and $\varsigma_{0}$.

Now, with these bounds on $m$ and $M$, one obtains that $\theta \in]-\infty, 1[$ and $\vartheta \in$ ] $-1,+\infty$ [. With these bounds on $\theta$ and $\vartheta$, one verifies that (4.2) holds.

\subsection{Construction of the solution $\bar{U}$}

Here, for writing purposes, the computation of $\bar{U}$ is decomposed into three steps: 1-construct $\tilde{U}=(\tilde{u}, \tilde{v}) ; 2$-compute $Z^{-}(\tilde{U}), Z^{+}(\tilde{U})$ and $\Gamma(\tilde{U}) ; 3$-compute $\bar{U}=(\bar{u}, \bar{v})$.

\section{Solution in short time $\tilde{U}$}

As in Section 2.3.2, a source $\tilde{\Sigma}=(\tilde{\sigma}, \tilde{\varsigma})$ constant along the (respective) characteristics is constructed, i.e. $\tilde{\sigma}$ (respectively $\tilde{\varsigma}$ ) is constant along $C_{-\mu}(x)\left(\right.$ resp. $\left.C_{-\nu}(x)\right)$ for all $x$, and equal to the value of $\sigma$ (resp. $\varsigma$ ) at the foot of the characteristic $C_{-\mu}(x, t)\left(\right.$ resp. $\left.C_{\nu}(x, t)\right)$. This yields

$$
\tilde{\sigma}(x, t)=\sigma_{0}(x+\mu t), \quad \tilde{\varsigma}(x, t)=\varsigma_{0}(x-\nu t) .
$$


Then, a field $\tilde{U}=(\tilde{u}, \tilde{v})=R(\tilde{\Sigma})$ is constructed

$$
\begin{aligned}
& \tilde{u}(x, t)=u_{0}(x+\mu t)+\sigma_{0}(x+\mu t) t, \\
& \tilde{v}(x, t)=v_{0}(x-\nu t)+\varsigma_{0}(x-\nu t) t .
\end{aligned}
$$

Computation of the boiling curve $\Gamma(\tilde{U})$

With this $\tilde{U}$, we compute $T$, the graph of which is $\Gamma(\tilde{U})$, and verify that $\Gamma(\tilde{U})$ is overcharacteristic.

Lemma 4.4. Suppose (4.2) holds. Then, there exists a unique $T \in W^{1, \infty}(\mathbb{R})$ such that for all $(x, t) \in \mathbb{R} \times \mathbb{R}^{+}$

$$
\left\{\begin{array}{lll}
h(\tilde{U})(x, t)<0 & \text { if and only if } & t<T(x), \\
h(\tilde{U})(x, t)=0 & \text { if and only if } & t=T(x), \\
h(\tilde{U})(x, t)>0 & \text { otherwise. } &
\end{array}\right.
$$

Furthermore, $T$ satisfies a.e. in $x \in \mathbb{R}$

$$
m \leq T^{\prime}(x) \leq M .
$$

Especially, $T$ satisfies (3.1) and its graph $\Gamma(\tilde{U})$ is overcharacteristic.

Proof. This proof is in three steps: 1-compute all possible pointwise values of $T$ considering $\sigma$ and $\varsigma$ constant; 2-verify that this $T$ satisfies (3.1); 3-compute $\Gamma(\tilde{U})$.

(1) Denote $\sigma^{-}=a, \sigma^{+}=b, \varsigma^{-}=c$ and $\varsigma^{+}=d$ and define

$$
u^{ \pm}(x, t)=u_{0}(x+\mu t)+t \sigma^{ \pm}, \quad v^{ \pm}(x, t)=v_{0}(x-\nu t)+t \varsigma^{ \pm} .
$$

Since $U_{0} \in\left(W^{1, \infty}(\mathbb{R})\right)^{2}$ and since $h$ is linear, $h\left(u^{\star}, v^{\otimes}\right) \in W^{1, \infty}\left(\mathbb{R} \times \mathbb{R}^{+}\right)$for all $\star= \pm$ and $\otimes= \pm$. Using an implicit function theorem (see e.g. [10] for a weak version) provides

$$
T^{\star, \otimes} \in W^{1, \infty}(\mathbb{R}), \text { s.t. } h\left(u^{\star}, v^{\otimes}\right)\left(x, T^{\star, \otimes}(x)\right)=0, \quad \star= \pm, \quad \otimes= \pm .
$$

(2) Differentiating a.e. the function $x \mapsto h\left(u^{\star}, v^{\otimes}\right)\left(T^{\star, \otimes}(x), x\right)$ leads to

$$
\begin{aligned}
\frac{d T^{\star, \otimes}}{d x}(x) & =-\frac{\partial_{x} h\left(u^{\star}, v^{\otimes}\right)\left(x, T^{\star, \otimes}(x)\right)}{\partial_{t} h\left(u^{\star}, v^{\otimes}\right)\left(x, T^{\star, \otimes}(x)\right)} \\
& =\frac{-\left[h_{1} u_{0}^{\prime}\left(x+\mu T^{\star, \otimes}(x)\right)+h_{2} v_{0}^{\prime}\left(x-\nu T^{\star, \otimes}(x)\right)\right]}{h_{1}\left[\mu u_{0}^{\prime}\left(x+\mu T^{\star, \otimes}(x)\right)+\sigma^{\star}\right]+\left[-\nu v_{0}^{\prime}\left(x-\nu T^{\star, \otimes}(x)\right)+\varsigma^{\otimes}\right]} .
\end{aligned}
$$

The denominator in (4.6) is non-zero according to (4.2). As in the proof of Proposition 3.3,

$$
(X, Y) \mapsto-\frac{h_{1} X+h_{2} Y}{h_{1}\left(\mu X+\sigma^{\star}\right)+h_{2}\left(-\nu Y+\varsigma^{\otimes}\right)}
$$




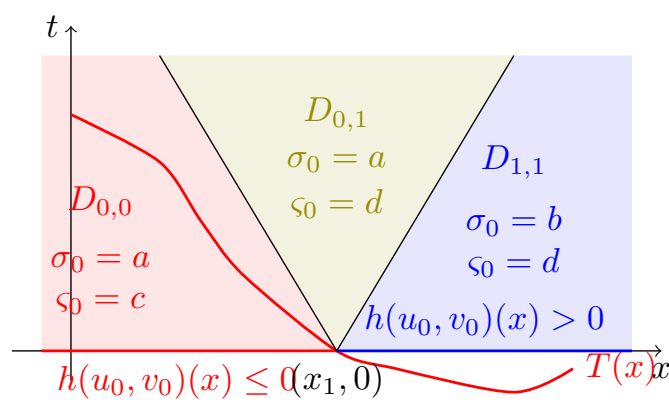

Fig. 8. Example of a function $T$ such that $T\left(x_{1}\right)=0$ and domains $D_{0,0}, D_{0,1}$ and $D_{1,1}$ separated by $C_{-\mu}\left(x_{1}\right)$ and $C_{\nu}\left(x_{1}\right)$.

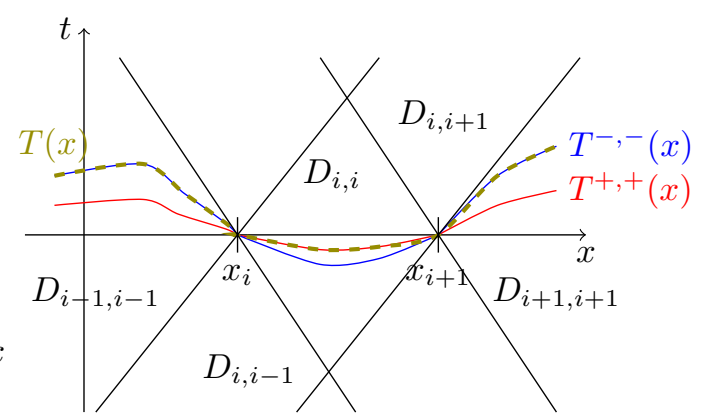

Fig. 9. Example of domains $D_{i, j}$ and functions $T^{++}, T^{--}$and $T$.

is strictly monotonic w.r.t. $\left.X \in\left[\inf u_{0}^{\prime}, \sup u_{0}^{\prime}\right] \subset\right] \sigma^{\star} / \mu,+\infty[$ and to $Y \in$ $\left.\left[\inf v_{0}^{\prime}, \sup v_{0}^{\prime}\right] \subset\right]-\infty, \varsigma^{\otimes} / \nu\left[\right.$. With the bounds $(4.2 \mathrm{~b})$ on $u_{0}^{\prime}$ and $(4.2 \mathrm{c})$ on $v_{0}^{\prime}$, the derivatives (4.6) satisfy (4.5).

(3) Suppose that the initial source $\Sigma_{0}=\left(\sigma_{0}, \varsigma_{0}\right)$ does not jump, then $\Sigma_{0}$ is constant equal to $(a, c)$ or $(b, d)$, and $T$ is either $T^{+,+}$or $T^{-,-}$, which both satisfy (4.5).

Suppose that $\Sigma_{0}$ jumps at the points $\left(x_{i}\right)_{i \in J}$ (potentially infinite numbered) such that $x_{i}<x_{i+1}$. By construction, $h(\tilde{U})$ is discontinuous across $C_{-\mu}\left(x_{i}\right)$ and $C_{\nu}\left(x_{i}\right)$ and $W^{1, \infty}$ elsewhere. Then, decompose the $(x, t)$-space into (see Fig. 8 and 9)

$$
D_{i, j}=\left\{\bigcup_{x \in] x_{i}, x_{i+1}[} C_{-\mu}(x)\right\} \cap\left\{\bigcup_{x \in] x_{j}, x_{j+1}[} C_{\nu}(x)\right\} .
$$

where $x_{\min J-1}=-\infty$ if $\min x_{i}>-\infty$ and $x_{\max J+1}=+\infty$ if $\max x_{i}<+\infty$.

Inside $D_{i, j}$, the source $\tilde{\Sigma}=(\tilde{\sigma}, \tilde{\varsigma})$ is constant, and $h(\tilde{U}) \in W^{1, \infty}\left(D_{i, j}\right)$ (from (4.3)). Since the $T^{\star, \otimes}$ satisfy (4.5), their graphs are overcharacteristic. And they pass at the points $\left(x_{i}, 0\right)$ for all $i \in J$. This provides for all $\star= \pm$ and $\otimes= \pm$

$$
\left\{\left(x, T^{\star, \otimes}(x)\right) \quad x \in \mathbb{R}\right\} \in \bigcup_{i} D_{i, i} .
$$

In each $D_{i, i}$, the value of $\tilde{U}$ is either $U^{+}$or $U^{-}$, then $T$ is also either $T^{+,+}$or $T^{-,-}$over $] x_{i}, x_{i+1}[$. They both satisfy (4.5) in each $] x_{i}, x_{i+1}[$ then $T$ satisfies $(4.5)$ over $\mathbb{R}$. And $h(\tilde{U}) \neq 0$ for all $(x, t) \in D_{i, j}$ for $i \neq j$.

Furthermore, using (4.2b) and (4.2c), $h\left(u^{\star}, v^{\otimes}\right)$ is strictly increasing along $C_{-\mu}(x)$ and $C_{\nu}(x)$ for all $x \in \mathbb{R}$. Thus it is negative for all $t<T^{\star, \otimes}(x)$ and positive for all $t>T^{\star, \otimes}(x)$. One deduces that $h(\tilde{U})$ has opposite signs on each side of $\Gamma(\tilde{U})$. 
Correction of the solution for long time $\bar{U}$

Computing $\bar{U}=R(\Sigma(\tilde{U}))$ from $(2.16,2.12)$ gives

$$
\begin{aligned}
& \bar{u}(x, t)=u_{0}(x+\mu t)+a \mathcal{T}(x, t)+b(t-\mathcal{T}(x, t)), \\
& \bar{v}(x, t)=v_{0}(x-\nu t)+c \mathfrak{T}(x, t)+d(t-\mathfrak{T}(x, t)), \\
& \mathcal{T}(x, t)=\int_{0}^{t} \mathbb{1}_{\mathbb{R}^{-}}(h(\tilde{u}, \tilde{v})(x+\mu(t-\tau), \tau)) d \tau, \\
& \mathfrak{T}(x, t)=\int_{0}^{t} \mathbb{1}_{\mathbb{R}^{-}}(h(\tilde{u}, \tilde{x})(x-\nu(t-\tau), \tau)) d \tau .
\end{aligned}
$$

where the integrals can be computed explicitly

$$
\begin{aligned}
& \mathcal{T}(x, t)= \begin{cases}0 & \text { if } \mathfrak{t}(x+\mu t)<0, \\
t & \text { if } 0<t<\mathfrak{t}(x+\mu t), \\
\mathfrak{t}(x+\mu t) & \text { otherwise, }\end{cases} \\
& \mathfrak{T}(x, t)= \begin{cases}0 & \text { if } \tau(x-\nu t)<0, \\
t & \text { if } 0<t<\tau(x-\nu t), \\
\tau(x-\nu t) & \text { otherwise. }\end{cases}
\end{aligned}
$$

Here, $\mathfrak{t}(x)$, resp. $\tau(x)$, is the time of intersection between $C_{-\mu}(x)$, resp. $C_{\nu}(x)$, and $\Gamma(\tilde{U})$. It yields

$$
\mathfrak{t}(x)=T\left((I d+\mu T)^{-1}(x)\right), \quad \tau(x)=T\left((I d-\nu T)^{-1}(x)\right) .
$$

Remark that if $T^{\prime}$ is bounded by (3.1), then $I d+\mu T$ and $I d-\nu T$ are strictly monotonic, thus bijective, and $(4.9 \mathrm{c})$ is well-defined.

For $\bar{U}=(\bar{u}, \bar{v})$ to be a generalized solution, there remains to prove that it satisfies (2.15).

Lemma 4.5. Suppose that (4.2) holds. Then, $\bar{U}=(\bar{u}, \bar{v})$ given by (4.8-4.9) satisfies

$$
\left(\partial_{t}-\mu \partial_{x}\right) h(\bar{U})>0, \quad\left(\partial_{t}+\nu \partial_{x}\right) h(\bar{U})>0 \quad \text { a.e. in }(x, t) \in \mathbb{R} \times \mathbb{R}^{+} .
$$

Proof. Compute

$$
\begin{aligned}
& \left(\partial_{t}-\mu \partial_{x}\right) h(\bar{U})(x, t)=h_{1}\left(\partial_{t}-\mu \partial_{x}\right) \bar{u}(x, t)+h_{2}\left(\partial_{t}-\mu \partial_{x}\right) \bar{v}(x, t), \\
& \left(\partial_{t}+\nu \partial_{x}\right) h(\bar{U})(x, t)=h_{1}\left(\partial_{t}+\nu \partial_{x}\right) \bar{u}(x, t)+h_{2}\left(\partial_{t}+\nu \partial_{x}\right) \bar{v}(x, t),
\end{aligned}
$$

where

$$
\begin{aligned}
& \left(\partial_{t}-\mu \partial_{x}\right) \bar{u}(x, t)=\left\{\begin{array}{lll}
a & \text { if } & (x, t) \in Z^{-}(\tilde{U}), \\
b & \text { if } & (x, t) \in Z^{+}(\tilde{U}),
\end{array}\right. \\
& \left(\partial_{t}+\nu \partial_{x}\right) \bar{v}(x, t)=\left\{\begin{array}{lll}
c & \text { if } & (x, t) \in Z^{-}(\tilde{U}), \\
d & \text { if } & (x, t) \in Z^{+}(\tilde{U}) .
\end{array}\right.
\end{aligned}
$$

Note that all the identities hold a.e. Using (4.9), the other terms read

$$
\begin{aligned}
\left(\partial_{t}-\mu \partial_{x}\right) \bar{v}(x, t) & =-(\mu+\nu) v_{0}^{\prime}(x-\nu t)+d+(c-d)\left(\partial_{t}-\mu \partial_{x}\right) \mathfrak{T}(x, t), \\
\left(\partial_{t}+\nu \partial_{x}\right) \bar{u}(x, t) & =(\mu+\nu) u_{0}^{\prime}(x+\mu t)+b+(a-b)\left(\partial_{t}+\nu \partial_{x}\right) \mathcal{T}(x, t),
\end{aligned}
$$




$$
\begin{aligned}
& \left(\partial_{t}+\nu \partial_{x}\right) \mathcal{T}(x, t)= \begin{cases}0 & \text { if } \mathfrak{t}(x+\mu t)<0, \\
1 & \text { if } 0<t<\mathfrak{t}(x+\mu t), \\
(\mu+\nu) \mathfrak{t}^{\prime}(x+\mu t) & \text { otherwise. }\end{cases} \\
& \left(\partial_{t}-\mu \partial_{x}\right) \mathfrak{T}(x, t)= \begin{cases}0 & \text { if } \tau(x-\nu t)<0, \\
1 & \text { if } 0<t<\tau(x-\nu t), \\
-(\mu+\nu) \tau^{\prime}(x-\nu t) & \text { otherwise, }\end{cases} \\
& \mathfrak{t}^{\prime}(x)=\frac{T^{\prime}}{1+\mu T^{\prime}}\left((I d+\mu T)^{-1}(x)\right), \quad \tau^{\prime}(x)=\frac{T^{\prime}}{1-\nu T^{\prime}}\left((I d-\nu T)^{-1}(x)\right), \\
& =\frac{1}{\frac{1}{T^{\prime}}+\mu}\left((I d+\mu T)^{-1}(x)\right), \quad=\frac{1}{\frac{1}{T^{\prime}}-\nu}\left((I d-\nu T)^{-1}(x)\right),
\end{aligned}
$$

From (4.5), $1+\mu T^{\prime}>0$ and $1-\nu T^{\prime}>0$ a.e. Then, where $T^{\prime}(x)=0$, one has $\mathfrak{t}^{\prime}(x)=0$ and $\tau^{\prime}(x)=0$, elsewhere

$$
\begin{aligned}
\mathfrak{t}^{\prime}(x) & =\frac{1}{\frac{1}{T^{\prime}}+\mu}\left((I d+\mu T)^{-1}(x)\right) \geq \frac{1}{\frac{1}{\sup T^{\prime}}+\mu} \geq \frac{M}{1+\mu M} \quad \text { a.e. } \\
\tau^{\prime}(x) & =\frac{1}{\frac{1}{T^{\prime}}-\nu}\left((I d-\nu T)^{-1}(x)\right) \leq \frac{1}{\frac{1}{\inf T^{\prime}}-\nu} \leq \frac{m}{1-\nu m} \quad \text { a.e. }
\end{aligned}
$$

Injecting these estimates in (4.10) leads to

$$
\begin{aligned}
& \left(\partial_{t}-\mu \partial_{x}\right) h(\bar{u}, \bar{v}) \geq h_{1} \min (a, b)+h_{2}\left[-(\mu+\nu) \sup v_{0}^{\prime}+\min (d, c, d+(c-d) \vartheta)\right], \\
& \left(\partial_{t}+\nu \partial_{x}\right) h(\bar{u}, \bar{v}) \geq h_{1}\left[(\mu+\nu) \inf u_{0}^{\prime}+\min (b, a, b+(a-b) \vartheta)\right]+h_{2} \min (c, d),
\end{aligned}
$$

the right-hand sides are strictly positive according to $(4.2 \mathrm{~b})$ and $(4.2 \mathrm{c})$, which gives the result.

From this lemma, one deduces that (2.20) holds and thus that $\bar{U}$ is a generalized solution.

Lemma 4.6. Suppose that (4.2) holds. Then, $\tilde{U}=(\tilde{u}, \tilde{v})$ given by (4.3) and $\bar{U}=$ $(\bar{u}, \bar{v})$ given by (4.8-4.9) satisfy

$$
\operatorname{sign}(h(\tilde{U}))=\operatorname{sign}(h(\bar{U})) .
$$

Proof. Consider first a sub-domain $D_{i, i}$ (from (4.7)), such that $h\left(U_{0}\right)(x)<0$ for all $(x, t) \in D_{i, i}$. In this set, $\operatorname{sign}(h(\tilde{U}))$ is given by (4.4).

Consider $(x, t) \in D_{i, i}$ such that $0<t<T(x)$ (see Fig. 9). Since $\Gamma(\tilde{U})$ is overcharacteristic, we have

$$
\forall \tau \in[0, T(x)[, \quad h(\tilde{U})(x+\mu \tau, t-\tau)<0 \quad \text { and } \quad h(\tilde{U})(x-\nu \tau, t-\tau)<0 .
$$

Comparing (4.3) and (4.8) gives $\tilde{U}(x, t)=\bar{U}(x, t)$, and $\operatorname{sign}(h(\tilde{U})(x, t))=$ $\operatorname{sign}(h(\bar{U})(x, t))$.

Now, use Lemma 4.5 for all other points. That is first for all $(x, t) \in D_{i, i}$ such that $t>T(x)$, then for all $(x, t) \in D_{i, i}$ such that $h\left(u_{0}, v_{0}\right)(x)>0$ and eventually for all $(x, t) \in D_{i, j}$ with $i<j$, one has $h(\bar{U})(x, t)>0$. Especially, $\operatorname{sign}(h(\tilde{U})(x, t))=$ $\operatorname{sign}(h(\bar{U})(x, t))$. 


\subsection{Uniqueness of the generalized solution}

The proof of uniqueness mimics the one of Lemma 4.6, but with the constraint (4.2) on the generalized solution $U$ instead of assuming that $U$ is given by the construction (4.8-4.9).

First let us state a regularity result.

Lemma 4.7. Consider $T \in W^{1, \infty}(] z, z+h[)$ such that for all $\left.x \in\right] z, z+h[$,

$$
\frac{-1}{\mu}<\lim _{\substack{y \rightarrow x \\ y<x}} T^{\prime}(y)<\frac{1}{\nu}, \quad \frac{-1}{\mu}<\lim _{\substack{y \rightarrow x \\ y>x}} T^{\prime}(y)<\frac{1}{\nu},
$$

and a generalized solution $U \in E$ such that (see Fig. 10)

$$
\forall(x, t) \in] z, z+h[\times[0, T(x)], \quad U(x, t)=\tilde{U}(x, t) \quad \text { and } \quad h(U)(x, t) \leq 0 .
$$

Then, $h(U)$ is continuous at $(x, T(x))$ for all $x \in] z, z+h[$.

Suppose furthermore that (4.2) holds and

$$
m \leq T^{\prime}(x) \leq M \text { for almost all } x \in \mathbb{R},
$$

where $m$ and $M$ are defined in (4.1). Then, for all $x \in] z, z+h[$ and all $\epsilon>0$ (small enough)

$$
\begin{aligned}
& h(U)(x-\mu \epsilon, T(x)+\epsilon)>h(U)(x, T(x)), \\
& h(U)(x+\nu \epsilon, T(x)+\epsilon)>h(U)(x, T(x)) .
\end{aligned}
$$

Remark 4.8. Since $T \in W^{1, \infty}(] z, z+h[)$, the limits in (4.13) are well-defined. Furthermore, the requirement (4.13) is weaker than (3.1) as the sup of $T^{\prime}$ could equal $\frac{1}{\nu}$ in $z+h$.

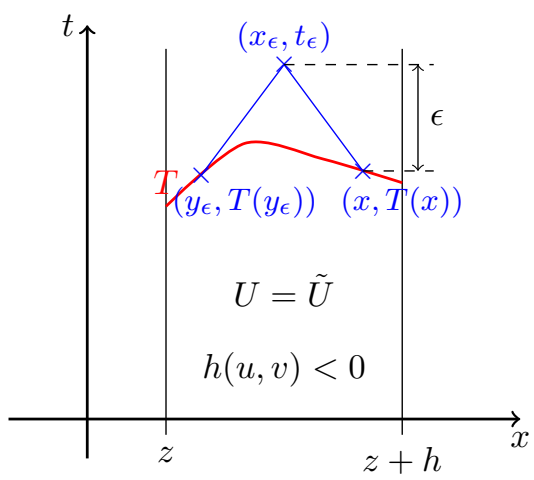

Fig. 10. Configuration for Lemma 4.7.

Proof. By hypothesis, $I d-\nu T$ and $I d+\mu T$ are bijective over $] z, z+h[$. 
Consider $x \in[z, z+h]$ and $\epsilon>0$ small enough. Define $t_{\epsilon}=T(x)+\epsilon$ and $\left.\left(x_{\epsilon}, y_{\epsilon}\right) \in\right] z, z+h\left[{ }^{2}\right.$ such that $\left(x_{\epsilon}, t_{\epsilon}\right) \in C_{\nu}\left(y_{\epsilon}, T\left(y_{\epsilon}\right)\right) \cap C_{-\mu}(x, T(x))$. This reads

$$
\left\{\begin{array}{l}
x_{\epsilon}=x-\mu \epsilon=y_{\epsilon}+\nu \varepsilon, \\
t_{\epsilon}=T(x)+\epsilon=T\left(y_{\epsilon}\right)+\varepsilon,
\end{array} \Rightarrow y_{\epsilon}=(I d-\nu T)^{-1}((I d-\nu T)(x)-(\mu+\nu) \epsilon) .\right.
$$

Thus, we impose the bound $0<\epsilon<\frac{(z-x)+\nu T(x)}{\mu+\nu}$ on $\epsilon$ in order that $y_{\epsilon}>z$.

Compute the integrals along $C_{-\mu}\left(x_{\epsilon}, t_{\epsilon}\right)$,

$$
\begin{aligned}
u\left(x_{\epsilon}, t_{\epsilon}\right) & =u(x, T(x))+\int_{T(x)}^{t_{\epsilon}} \sigma(u, v)(x-\mu \tau, \tau) d \tau \\
& =\tilde{u}(x, T(x))+\check{\sigma} \epsilon,
\end{aligned}
$$

where

$$
\check{\sigma}:=\frac{1}{\epsilon} \int_{T(x)}^{T(x)+\epsilon} \sigma(u, v)(x-\mu \tau, \tau) d \tau \in[a, b] .
$$

Similarly, along $C_{\nu}\left(x_{\epsilon}, t_{\epsilon}\right)$,

$$
\begin{aligned}
v\left(x_{\epsilon}, t_{\epsilon}\right) & =v\left(y_{\epsilon}, T\left(y_{\epsilon}\right)\right)+\int_{T\left(y_{\epsilon}\right)}^{T(x)+\epsilon} \varsigma(u, v)\left(y_{\epsilon}+\nu \tau, \tau\right) d \tau \\
& =\tilde{v}\left(y_{\epsilon}, T\left(y_{\epsilon}\right)\right)+\check{\varsigma} \epsilon+\hat{\varsigma} \int_{y_{\epsilon}}^{x} T^{\prime}(\xi) d \xi,
\end{aligned}
$$

where

$\check{\varsigma}:=\frac{1}{\epsilon} \int_{T(x)}^{T(x)+\epsilon} \varsigma(u, v)\left(y_{\epsilon}+\nu \tau, \tau\right) d \tau, \quad \hat{\varsigma}:=\frac{1}{T(x)-T\left(y_{\epsilon}\right)} \int_{T\left(y_{\epsilon}\right)}^{T(x)} \varsigma(u, v)\left(y_{\epsilon}+\nu \tau, \tau\right) d \tau$,

such that $(\check{\varsigma}, \hat{\varsigma}) \in[c, d]^{2}$. Here, one rewrites

$$
\begin{aligned}
\tilde{v}\left(y_{\epsilon}, T\left(y_{\epsilon}\right)\right) & =\tilde{v}(x, T(x))-\int_{y_{\epsilon}}^{x} \frac{d}{d \xi} \tilde{v}(\xi, T(\xi)) d \xi \\
& =\tilde{v}(x, T(x))-\int_{y_{\epsilon}}^{x}\left[\left(1-\nu T^{\prime}(\xi)\right) v_{0}^{\prime}(\xi-\nu T(\xi))+c T^{\prime}(\xi)\right] d \xi .
\end{aligned}
$$

This leads to

$$
\begin{aligned}
D_{\epsilon} & :=\frac{h(u, v)\left(x_{\epsilon}, t_{\epsilon}\right)-h(u, v)(x, T(x))}{\epsilon} \\
& =h(\check{\sigma}, \check{\varsigma})-\frac{h_{2}}{\epsilon} \int_{y_{\epsilon}}^{x}\left[\left(1-\nu T^{\prime}(\xi)\right) v_{0}^{\prime}(\xi-\nu T(\xi))+(c-\hat{\varsigma}) T^{\prime}(\xi)\right] d \xi .
\end{aligned}
$$

Then, one obtains from (4.15)

$$
\begin{aligned}
\lim _{\epsilon \rightarrow 0} D_{\epsilon} & =h(\check{\sigma}, \check{\zeta})-\frac{h_{2}(\mu+\nu)}{1-\nu T^{\prime}\left(x^{-}\right)}\left[\left(1-\nu T^{\prime}\left(x^{-}\right)\right) v_{0}^{\prime}\left(x^{-}-\nu T\left(x^{-}\right)\right)+(c-\hat{\varsigma}) T^{\prime}\left(x^{-}\right)\right] \\
& =h(\check{\sigma}, \check{\zeta})-h_{2}(\mu+\nu)\left[v_{0}^{\prime}\left(x^{-}-\nu T\left(x^{-}\right)\right)+(c-\hat{\varsigma}) \frac{T^{\prime}\left(x^{-}\right)}{1-\nu T^{\prime}\left(x^{-}\right)}\right]
\end{aligned}
$$


where $f\left(x^{-}\right)=\lim _{\substack{y \rightarrow x \\ y<x}} f(y)$. By hypothesis, these derivatives exist a.e. in $\left.x \in\right] z, z+h[$. Thus these limits exist, and $1-\nu T^{\prime}\left(x^{-}\right)>0$. Especially, $\lim _{\epsilon \rightarrow 0} D_{\epsilon}$ exists and is finite. Thus $h(U)$ is continuous at $(x, T(x))$ for all $x \in \mathbb{R}$.

Assume furthermore that (4.2) and (4.14) hold. Rewrite

$$
\epsilon=\frac{1}{\mu+\nu} \int_{(I d-\nu T)(x)-(\mu+\nu) \epsilon}^{(I d-\nu T)(x)} d y=\frac{1}{\mu+\nu} \int_{y_{\epsilon}}^{x}\left(1-\nu T^{\prime}\right)(\xi) d \xi
$$

Then

$$
\begin{aligned}
D_{\epsilon} & =\int_{y_{\epsilon}}^{x} \frac{\left(1-\nu T^{\prime}\right)(\xi)}{\mu+\nu}\left[h(\check{\sigma}, \check{\zeta})-h_{2}(\mu+\nu)\left(v_{0}^{\prime}(\xi-T(\xi))+(c-\check{\varsigma}) \frac{T^{\prime}}{1-\nu T^{\prime}}(\xi)\right)\right] d \xi \\
& \geq \int_{y_{\epsilon}}^{x} \frac{\left(1-\nu T^{\prime}\right)(\xi)}{\mu+\nu} h\left(\check{\sigma}, \check{\varsigma}-(\mu+\nu) \sup v_{0}^{\prime}+(c-\check{\varsigma}) \vartheta\right) d \xi>0 .
\end{aligned}
$$

Similar computations hold on the other characteristics.

Definition 4.9. Following [6], define the cone of dependence (see Fig. 3)

$C(x, t):=\left\{(y, \tau) \in \mathbb{R}^{+} \times \mathbb{R} \quad\right.$ s.t. $\quad \tau \in[0, t]$ and $\left.y \in[x-\nu(t-\tau), x+\mu(t-\tau)]\right\}$.

Considering a generalized solution $U=(u, v) \in E$, define (see Fig. 11)

$D^{-}(U):=\left\{(x, t) \in \mathbb{R} \times \mathbb{R}^{+} \quad\right.$ s.t. $h(u, v)(y, \tau) \leq 0 \quad$ a.e. in $\left.(y, \tau) \in C(x, t)\right\} \subset Z^{-}(U)$.

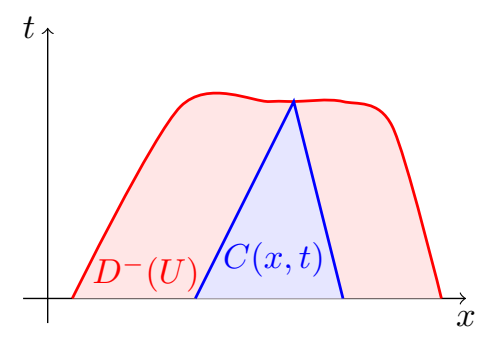

Fig. 11. Example of a set $D^{-}(U)$ (pale red) and a cone of dependence $C(x, t)$ (pale blue) for a point $(x, t) \in D^{-}(U)$.

Now, let us show that $U$ is equal $\bar{U}$ in $Z^{-}(\tilde{U})$.

Lemma 4.10. Suppose that (4.2) holds and consider a generalized solution $U \in E$. Then,

$$
D^{-}(U)=Z^{-}(\tilde{U}) .
$$

Especially, $U(x, t)=\bar{U}(x, t)$ for all $(x, t) \in Z^{-}(\tilde{U})$. 
Proof. Any generalized solution $U=(u, v) \in E$ is of the form (2.15) which rewrites (4.8) for some functions $(\mathcal{T}, \mathfrak{T})$. Since $U_{0} \in\left(W^{1, \infty}(\mathbb{R})\right)^{2}$ and $U \in E$, we obtain that $(\mathcal{T}, \mathfrak{T}) \in E$.

Since $U$ satisfies (2.15), then $U(x, t)=\tilde{U}(x, t)$ for almost all $(x, t) \in D^{-}(U)$. Thus

$$
D^{-}(U) \subset Z^{-}(\tilde{U})
$$

By construction,

$$
D^{-}(U)=\left\{(x, t) \in \mathbb{R} \times \mathbb{R}^{+} \text {s.t. } x \in\left[x_{2 i}, x_{2 i+1}\right] \quad \text { and } t \in\left[0, T_{i}(x)\right]\right\},
$$

where the $x_{j}$ are such that $h\left(U_{0}\right)(x) \leq 0$ a.e. on $\left[x_{2 i}, x_{2 i+1}\right]$ and the $T_{i} \in W^{1, \infty}(\mathbb{R})$ are such that

$0 \leq T_{i}(x) \quad \forall x \in\left[x_{2 i}, x_{2 i+1}\right] \quad$ and $\quad \frac{-1}{\mu} \leq T_{i}^{\prime}(x) \leq \frac{1}{\nu}$ for almost all $x \in\left[x_{2 i}, x_{2 i+1}\right]$, and $\left(x, T_{i}(x)+\epsilon\right) \notin D^{-}(U)$ for all $\epsilon>0$. According to (4.18), $T_{i} \leq T$ a.e.

First, the graph of $T_{i}$ is shown to be overcharacteristic in a subset of $\left[x_{2 i}, x_{2 i+1}\right]$, then in the entire interval $\left[x_{2 i}, x_{2 i+1}\right]$, and finally it is shown to be $\Gamma(\tilde{U})$, i.e. $T_{i}(x)=$ $T(x)$ for all $x \in\left[x_{2 i}, x_{2 i+1}\right]$.

(1) Suppose first that $T_{i}^{\prime}\left(x_{2 i}\right)=\frac{1}{\nu}$ (see Fig. 12). Then, $T_{i}^{\prime}\left(x_{2 i}\right)>T^{\prime}\left(x_{2 i}\right)$ and by continuity of $T_{i}$ and $T$, there exists $\epsilon>0$ such that $T_{i}(x)>T(x)$ over $x \in\left[x_{2 i}, x_{2 i}+\epsilon\right]$. By definition of $T_{i}$,

$$
\forall x \in\left[x_{2 i}, x_{2 i}+\epsilon\right], \quad\left(x, T_{i}(x)\right) \in C\left(x, T_{i}(x)\right) \subset D^{-}(\tilde{U}) .
$$

However, one verifies geometrically that

$$
\forall x \in\left[x_{2 i}, x_{2 i}+\epsilon\right], \quad C\left(x, T_{i}(x)\right) \backslash Z^{-}(\tilde{U}) \neq \emptyset,
$$

which contradicts (4.18). Then $T_{i}^{\prime}\left(x_{2 i}\right)<\frac{1}{\nu}$. Thus there exists $\epsilon>0$ such that $0<T_{i}^{\prime}(x)<\frac{1}{\nu}$ for all $x \in\left[x_{2 i}, x_{2 i}+\epsilon\right]$. Similarly, one finds $0>T_{i}^{\prime}(x)>\frac{-1}{\mu}$ for all $x \in\left[x_{2 i+1}-\epsilon, x_{2 i+1}\right]$.

(2) Suppose now that $T_{i}^{\prime}(x)<\frac{1}{\nu}$ for all $x \in\left[x_{2 i}, y\right.$ [ and $T_{i}^{\prime}(y)=\frac{1}{\nu}$ (see Fig. 12). Especially, $I d-\nu T$ is bijective over $\left[x_{2 i}, y\right]$.

Since $T^{\prime}$ cannot reach such a value and $T_{i} \leq T$, there exists $\epsilon>0$ such that $T_{i}(x)<T(x)$ for all $x \in[y-\epsilon, y]$.

Lemma 4.7 provides the continuity of $h(U)$ along the graph of $T_{i}$. Especially

$$
h(U)\left(x, T_{i}(x)\right)=h(\tilde{U})\left(x, T_{i}(x)\right)<0 \quad \forall x \in[y-\epsilon, y] .
$$

By continuity, one can find a neighbourhood of $\left(x, T_{i}(x)\right)$ on which $h(U)$ is strictly negative (in gray on Fig. 12). Inside this neighbourhood, one may find a point $(z, \tau)$ with $\tau>T_{i}(z)$ such that $h(U)$ is strictly negative over all $C(z, \tau)$. This implies that $C(z, \tau) \subset D^{-}(U)$, which contradicts (4.19).

Thus, $T_{i}^{\prime}(x)<\frac{1}{\nu}$ for all $x \in\left[x_{2 i}, x_{2 i+1}\right]$. Similar computations give $T_{i}^{\prime}(x)>$ $\frac{-1}{\mu}$ for all $x \in\left[x_{2 i}, x_{2 i+1}\right]$ and thus $T_{i}$ has an overcharacteristic graph. 
(3) Finally, suppose that there exists $y \in\left[x_{2 i}, x_{2 i+1}\right]$ such that $T_{i}(x)<T(x)$. Applying again Lemma 4.7 along the graph of $T_{i}$ provides the existence of a neighbourhood of $\left(y, T_{i}(y)\right)$ on which $h(u, v)<0$. This neighbourhood contains $C(z, \tau) \backslash D^{-}(U)$ for some $(z, \tau)$ satisfying $\tau>T_{i}(z)$ which contradicts the definition of $D^{-}(U)$. Thus $T(x)=T_{i}(x)$ for all $x \in\left[x_{2 i}, x_{2 i+1}\right]$.
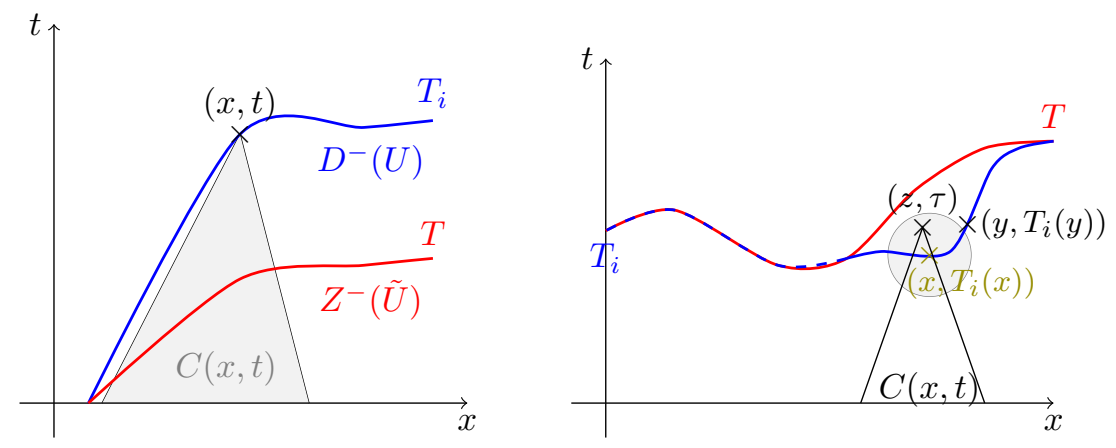

Fig. 12. Configuration at steps 1 (left) and 2 (right) of the proof of Lemma 4.10.

Finally, uniqueness is obtained in all the $(x, t)$-space: from Lemma $4.10, \bar{U}$ is the unique generalized solution in $Z^{-}(\tilde{U})$. Furthermore,

$$
Z^{-}(\tilde{U})=\left\{(x, t) \in \mathbb{R} \times \mathbb{R}^{+} \text {s.t. } t \leq T(x)\right\},
$$

where the graph of $T \in W^{1, \infty}(\mathbb{R})$ is overcharacteristic. Then Lemma 4.7 provides the positivity of $h(U)$ for all $(x, t) \notin Z^{-}(\tilde{U})$, and one identifies $\bar{U}$ to $U$ through (4.8).

One easily verifies that the unique generalized solution $\bar{U}$ depends continuously on $a, b, c, d$ and $U_{0}$ inside the set defined by (4.2). Especially, the problem (2.15) is well-posed in the sense of Hadamard under these constraints.

\section{Solution in the regular subcharacteristic case (Theorem 3.5)}

Again, the proof of Theorem 3.5 is based on Lemma 5.2 below.

\subsection{Notations and precise statement}

Let us introduce notations similar to those of the preceding section.

Notation 5.1. Define

$$
\Xi(y, z):=\frac{-\left[h_{1}\left(\mu u_{0}^{\prime}+\sigma_{0}\right)(y)+h_{2}\left(-\nu v_{0}^{\prime}+\varsigma_{0}\right)(z)\right]}{h_{1} u_{0}^{\prime}(y)+h_{2} v_{0}^{\prime}(z)}=\frac{1}{\Theta(y, z)},
$$




$$
\begin{array}{rlrl}
\mathfrak{m} & :=\inf _{(y, z) \in \mathbb{R}^{2}} \Xi(y, z), & \mathfrak{M}:=\sup _{(y, z) \in \mathbb{R}^{2}} \Xi(y, z), \\
\theta:=\frac{\mu+\nu}{\mu+\mathfrak{m}}, & \vartheta:=\frac{\mu+\nu}{\nu-\mathfrak{M}} .
\end{array}
$$

Lemma 5.2. Suppose that $U_{0} \in\left(W^{1, \infty}(\mathbb{R})\right)^{2}$ is such that

$$
\begin{array}{cc}
-\mu<\mathfrak{m}, & \mathfrak{M}<\nu, \\
h_{1}(\mu+\nu) \sup _{x}\left(-u_{0}^{\prime}\right)<h_{1} \min (a, b, a+(b-a) \theta)+h_{2} \min (c, d), & \\
h_{2}(\mu+\nu) \inf _{x}\left(v_{0}^{\prime}\right)>h_{1} \max (a, b) & +h_{2} \max (c, d, d+(c-d) \vartheta) .
\end{array}
$$

Then, there exists a unique generalized solution $U \in E$ to (1.2)

Remark 5.3. Again, the coefficients $\mathfrak{m}$ and $\mathfrak{M}$ correspond to the bounds on the derivative $X^{\prime}$ of the function $X$ the graph of which is $\Gamma(\tilde{U})$.

Proof of Theorem 3.5. This is obtained by a straightforward adaptation of the proof of Theorem 3.3.

\subsection{Construction of the generalized solution}

The function $\tilde{U}=(\tilde{u}, \tilde{v})$ has the same definition (4.3) as in the last section.

Boiling curve $\Gamma(\tilde{U})$

One verifies that $\Gamma(\tilde{U})$ is subcharacteristic.

Lemma 5.4. Suppose (5.2) holds. Then, there exists a unique $X \in W^{1, \infty}(\mathbb{R})$ such that for all $(x, t) \in \mathbb{R} \times \mathbb{R}^{+}$

$$
\left\{\begin{array}{l}
h(\tilde{U})(x, t)<0 \quad \Leftrightarrow \quad x<X(t), \\
h(\tilde{U})(x, t)=0 \quad \Leftrightarrow \quad x=X(t), \\
h(\tilde{U})(x, t)>0 \quad \Leftrightarrow \quad x>X(t) .
\end{array}\right.
$$

Furthermore, $X$ satisfies for almost all $t \in \mathbb{R}^{+}$

$$
\mathfrak{m} \leq X^{\prime}(t) \leq \mathfrak{M}
$$

Especially, $X$ satisfies (3.2) and its graph $\Gamma(\tilde{U})$ is subcharacteristic.

Remark 5.5. This case corresponds to the cases $T^{\prime}(x)>\frac{1}{\nu}$ and $T^{\prime}(x)<\frac{-1}{\mu}$ (see Fig. 13).

Proof. First, using the hypothesis (5.2), one computes

$$
\begin{aligned}
\partial_{x} h\left(U_{0}\right)(x) & =h_{1} u_{0}^{\prime}(x)+h_{2} v_{0}^{\prime}(x) \\
& >\frac{h_{1}(\max (a, b)-\min (a, b))+h_{2}(\max (c, d)-\min (c, d))}{\mu+\nu}>0 .
\end{aligned}
$$


Thus there exists one unique point where $h\left(U_{0}\right)$ changes sign.

Reproducing the computations of the proof of Lemma 4.4 (with the same notations) leads to

$$
\begin{aligned}
& \left(\partial_{t}-\mu \partial_{x}\right) h\left(u^{\star}, v^{\otimes}\right)(x, t) \leq-h_{2}(\mu+\nu) \inf v_{0}^{\prime}+h_{1} \max (a, b)+h_{2} \max (c, d)<0, \\
& \left(\partial_{t}+\nu \partial_{x}\right) h\left(u^{\star}, v^{\otimes}\right)(x, t) \geq h_{1}(\mu+\nu) \inf u_{0}^{\prime}+h_{1} \min (a, b)+h_{2} \min (c, d)>0 .
\end{aligned}
$$

The implicit function theorem provides again the existence of functions $X^{\star, \otimes} \in$ $W^{1, \infty}\left(\mathbb{R}^{+}\right)$such that $h\left(\bar{u}^{\star}, \bar{v}^{\otimes}\right)\left(X^{\star, \otimes}(t), t\right)=0$. Differentiating it with respect to $t$ leads to

$$
X^{\star, \otimes}(t)=\frac{-\left[h_{1}\left(\mu u_{0}^{\prime}\left(X^{\star, \otimes}(t)+\mu t\right)+\sigma^{\star}\right)+h_{2}\left(-\nu v_{0}^{\prime}\left(X^{\star, \otimes}(t)-\nu t\right)+\varsigma^{\otimes}\right)\right]}{h_{1} u_{0}^{\prime}\left(X^{\star, \otimes}(t)+\mu t\right)+h_{2} v_{0}^{\prime}\left(X^{\star, \otimes}(t)-\nu t\right)} .
$$

The inf and sup values are obtained for the inf and sup values of $u_{0}^{\prime}$ and $v_{0}^{\prime}$ and these estimates show that $X^{\star, \otimes}$ satisfies (5.4) for all $\star= \pm$ and $\otimes= \pm$.

Finally, one identifies $X=X^{-,+}$matching the signs of $h$ at $t=0$.
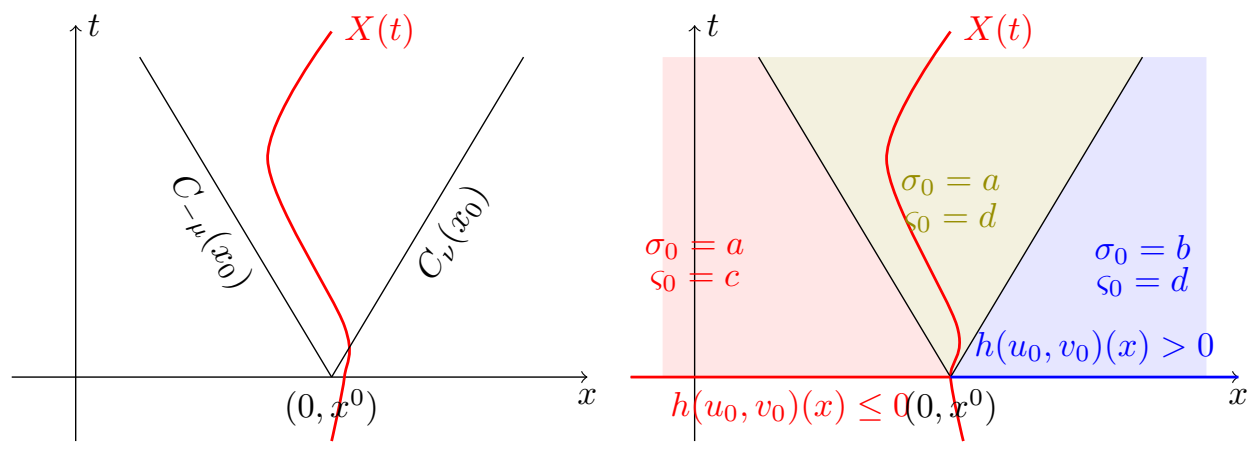

Fig. 13. Representation of $\Gamma(\tilde{U})$ (left), and of different zones where $h(\tilde{u}, \tilde{v})$ is linear (right).

Correction of the solution for long time $\bar{U}$

From (4.4), $Z^{+}(\tilde{U})$ is on the right of $Z^{-}(\tilde{U})$, then $\bar{U}$ is given by (4.8) where $\mathcal{T}$ and $\mathfrak{t}$ are

$$
\begin{aligned}
& \mathcal{T}(x, t)= \begin{cases}t & \text { if } \mathfrak{t}(x+\mu t)<0, \\
0 & \text { if } 0<t<\mathfrak{t}(x+\mu t), \\
t-\mathfrak{t}(x+\mu t) & \text { otherwise, }\end{cases} \\
& \mathfrak{T}(x, t)= \begin{cases}0 & \text { if } \tau(x-\nu t)<0, \\
t & \text { if } 0<t<\tau(x-\nu t), \\
\tau(x-\nu t) & \text { otherwise, }\end{cases}
\end{aligned}
$$


where $\mathfrak{t}(x)$, resp. $\tau(x)$, is the time of intersection between $C_{-\mu}(x)$, resp. $C_{\nu}$, and $\Gamma(\tilde{U})$. It reads

$$
\mathfrak{t}(x)=(X+\mu I d)^{-1}(x), \quad \tau(x)=(X-\nu I d)^{-1}(x) .
$$

Again, $h(\bar{U})$ is shown to have the same sign as $h(\tilde{U})$ by exploiting its monotonicity along the characteristics.

Lemma 5.6. Suppose that (5.2) holds. Then, $\bar{U}=(\bar{u}, \bar{v})$ given by $(4.8,5.6)$ satisfies

$$
\left(\partial_{t}-\mu \partial_{x}\right) h(\bar{U})<0, \quad\left(\partial_{t}+\nu \partial_{x}\right) h(\bar{U})>0 \quad \text { a.e. in }(x, t) \in \mathbb{R} \times \mathbb{R}^{+},
$$

Proof. The formula (4.10) holds in the present case. Then,

$$
\begin{aligned}
& \left(\partial_{t}-\mu \partial_{x}\right) \mathfrak{T}(x, t)= \begin{cases}0 & \text { if } \tau(x-\nu t)<0, \\
1 & \text { if } 0<t<\tau(x-\nu t), \\
-(\mu+\nu) \tau^{\prime}(x-\nu t) & \text { otherwise, }\end{cases} \\
& \left(\partial_{t}+\nu \partial_{x}\right) \mathcal{T}(x, t)= \begin{cases}1 & \text { if } \mathfrak{t}(x+\mu t)<0, \\
0 & \text { if } 0<t<\mathfrak{t}(x+\mu t), \\
1-(\mu+\nu) \mathfrak{t}^{\prime}(x+\mu t) & \text { otherwise. }\end{cases}
\end{aligned}
$$

Using (5.6), one has

$$
\mathfrak{t}^{\prime}(x)=\frac{1}{X^{\prime}\left((X+\mu I d)^{-1}(x)\right)+\mu}, \quad \boldsymbol{\tau}^{\prime}(x)=\frac{1}{X^{\prime}\left((X-\nu I d)^{-1}(x)\right)-\nu} .
$$

re-injecting this in (4.10) and using the estimates (5.4) on $X^{\prime}$ and those (5.2b-5.2c) on $U_{0}$ provides the result.

\subsection{Uniqueness of the generalized solution}

Again there remains to identify the unique solution $U$ to $\bar{U}$ given by $(4.8,5.6)$. This is obtained by adapting the proof of Section 5.3.

\section{Solution of the Riemann problem (Theorem 3.7)}

The proof of Theorem 3.7 is based on Lemma 6.1 below. The solution is easily found in the left domain $D_{L}=\left\{(x, t) \in \mathbb{R} \times \mathbb{R}^{+}\right.$s.t. $\left.x \leq-\mu t\right\}$ and in the right domain $D_{R}=\left\{(x, t) \in \mathbb{R} \times \mathbb{R}^{+}\right.$s.t. $\left.x>\nu t\right\}$. In the middle domain, its construction is adapted from the steps of Subsection 2.3.1.

\subsection{Notations and precise statement}

The following requirements are slightly less restrictive than (3.6) and sufficient to successfully construct a solution that provides a boiling line $\Gamma^{*}$ overcharacteristic in the middle domain $D_{*}$. 
Lemma 6.1. Suppose that

$$
\begin{gathered}
\min _{\tilde{\sigma}=a, b} \min _{\tilde{\varsigma}=c, d} h(\tilde{\sigma}, \tilde{\varsigma})>0, \\
\min _{\tilde{\sigma}=a, b} \frac{h(\tilde{\sigma}, d)}{h(\tilde{\sigma}, c)} \min _{\tilde{\varsigma}=c, d} h(a, \tilde{\varsigma})>h_{1}(a-b), \\
\min _{\tilde{\sigma}=a, b} h(\tilde{\sigma}, c) \min _{\tilde{\varsigma}=c, d} \frac{h(b, \tilde{\varsigma})}{h(a, \tilde{\varsigma})}>h_{2}(c-d) .
\end{gathered}
$$

Then, for all $\left(U_{L}^{0}, U_{R}^{0}\right) \in \mathbb{R}^{4}$, there exists a unique generalized solution to (1.2,3.5).

This result implies Theorem 3.7.

Proof of Theorem 3.7. Recall that $h_{1}>0$ and $h_{2}>0$. If (3.6) holds, then

$$
\min _{\tilde{\sigma}=a, b} \min _{\tilde{\varsigma}=c, d} h(\tilde{\sigma}, \tilde{\varsigma})=h(a, c)>0 .
$$

Furthermore, $h_{1}(a-b)<0$ and $h_{2}(c-d)<0$, while

$$
\begin{aligned}
& \min _{\tilde{\sigma}=a, b} \frac{h(\tilde{\sigma}, d)}{h(\tilde{\sigma}, c)} \min _{\tilde{\varsigma}=c, d} h(a, \tilde{\varsigma})>\frac{h(a, d)}{h(b, c)} h(a, c)>0>h_{1}(a-b), \\
& \min _{\tilde{\sigma}=a, b} h(\tilde{\sigma}, c) \min _{\tilde{\zeta}=c, d} \frac{h(b, \tilde{\varsigma})}{h(a, \tilde{\varsigma})}>h(a, c) \frac{h(b, c)}{h(a, d)}>0>h_{2}(c-d) .
\end{aligned}
$$

Thus, (6.1) holds and Lemma 6.1 provides the result.

The proof of Lemma 6.1 is restricted to the case where the Riemann data satisfy

$$
h\left(u_{L}^{0}, v_{L}^{0}\right)<0, \quad h\left(u_{R}^{0}, v_{R}^{0}\right)<0, \quad h\left(u_{R}^{0}, v_{L}^{0}\right)<0 .
$$

However it extends easily to general Riemann data. As illustrated below, (6.2) corresponds to the case where $h(U)$ changes sign in every domains $D_{L}, D^{*}$ and $D_{R}$, especially

$$
\begin{gathered}
\sigma\left(u_{L}^{0}, v_{L}^{0}\right)=\sigma\left(u_{R}^{0}, v_{R}^{0}\right)=\sigma\left(u_{R}^{0}, v_{L}^{0}\right)=a, \\
\varsigma\left(u_{L}^{0}, v_{L}^{0}\right)=\varsigma\left(u_{R}^{0}, v_{R}^{0}\right)=\varsigma\left(u_{R}^{0}, v_{L}^{0}\right)=c .
\end{gathered}
$$

\subsection{Solution in the left and right domains $D_{L}$ and $D_{R}$}

Denote $S=L, R$ the generic index associated to a side domain $D_{L}$ or $D_{R}$. A space independent solution is computed in a straightforward way.

Lemma 6.2. Suppose that $h(a, c) h(b, d)>0$. Then, there exists a unique generalized solution $U=(u, v)$ over $D_{S}$. Furthermore, $U$ does not depend on $x$ and satisfies 
for all $(x, t) \in D_{S}$

$$
\begin{aligned}
u(x, t)=u_{S}(t) & = \begin{cases}u_{S}^{0}+a t & \text { if } 0<t<\tau_{S}, \\
u_{S}^{0}+a \tau_{S}+b\left(t-\tau_{S}\right) & \text { if } \tau_{S}<t,\end{cases} \\
v(x, t)=v_{S}(t) & =\left\{\begin{array}{ll}
v_{S}^{0}+c t & \text { if } 0<t<\tau_{S}, \\
v_{S}^{0}+c \tau_{S}+d\left(t-\tau_{S}\right) & \text { if } \tau_{S}<t .
\end{array},\right. \\
\tau_{S} & =\frac{-h\left(u_{S}^{0}, v_{S}^{0}\right)}{h(a, c)},
\end{aligned}
$$

where $u_{S}$ and $v_{S}$ are defined by (6.4) below.

Proof. Define $u_{S}$ and $v_{S}$ such that

$$
u_{S}^{\prime}(t)=\sigma\left(u_{S}, v_{S}\right)(t), \quad v_{S}^{\prime}(t)=\varsigma\left(u_{S}, v_{S}\right)(t), \quad u_{S}(0)=u_{S}^{0}, \quad v_{S}(0)=v_{S}^{0} .
$$

One computes

$$
\frac{d}{d t} h\left(U_{S}\right)(t)=\left\{\begin{array}{lll}
h(a, c) & \text { if } & h\left(U_{S}\right)(t) \leq 0, \\
h(b, d) & \text { if } \quad h\left(U_{S}\right)(t)>0,
\end{array} \quad h\left(U_{S}\right)(0)=h\left(U_{S}^{0}\right),\right.
$$

which has a unique Carathodory solution according to Proposition 2.2 and (6.1a). One verifies that $(x, t) \mapsto U_{S}(t)$ is a generalized solution over $D_{S}$. The uniqueness is obtained by adapting the proof of Lemma 4.10.

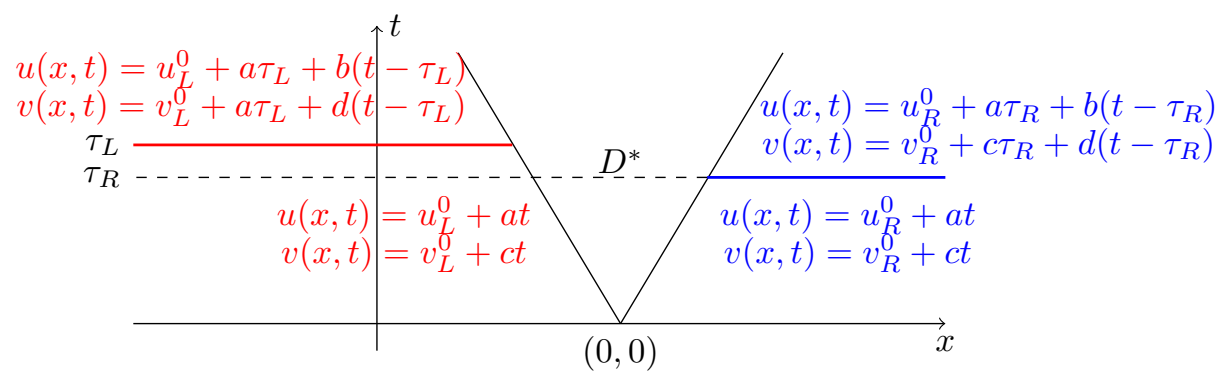

Fig. 14. Example of a solution $U=(u, v)$ in $D_{L}$ and $D_{R}$.

\subsection{Solution in the middle domain $D^{*}$}

Now, the solution (6.3) is prolonged in the middle domain $D^{*}$. As illustrated on Fig. 1 (bottom) and in the numerical example of Section 6.5 below, $h(u, v)$ may switch sign several times along a characteristic curve, due to the discontinuities of $u$, resp. $v$, across $C_{-\mu}(0)$, resp. $C_{\nu}(0)$. For this purpose, and since $U$ is already known in $D_{L}$ and $D_{R}$, the construction of $\tilde{U}$ is restricted inside $D^{*}$ and is constructed using continuity of $u$ along $C_{\nu}(0)$ and of $v$ along $C_{-\mu}(0)$. 
The construction of the solution follows the same guidelines as before, but is adapted to take into account boundary conditions along $C_{-\mu}(0)$ and $C_{\nu}(0)$.

Solution in short time $\tilde{U}$ in $D^{*}$

Consider the short time solution $\tilde{U}=(\tilde{u}, \tilde{v})$ inside $D^{*}$. The solution $\tilde{u}$ is continuous through $C_{\nu}(0)$ and along the characteristics $C_{-\mu}(x, t)$ the feet of which lie on $\{x>$ $0\}$. At such positions, $\tilde{u}(x, 0)=u_{R}^{0}$. Similarly, $\tilde{v}$ is continuous through $C_{-\mu}(0)$ and along the characteristics $C_{\nu}(x, t)$ the feet of which lie on $\{x<0\}$. At such positions, $\tilde{v}(x, 0)=v_{L}^{0}$. Since $h\left(u_{R}^{0}, v_{L}^{0}\right)<0$ is strictly negative and exploiting the regularity of the solution, $h(\tilde{U})$ is expected to remain negative for small enough time.

For these reasons, we fix the source term $\tilde{\Sigma}=(a, c)$ and replace the initial conditions by conditions along $C_{\nu}(0)$ and $C_{-\mu}(0)$. In other words, we replace the problem $(2.18)$ for $\tilde{U}=(\tilde{u}, \tilde{v})$ by

$$
\begin{aligned}
\partial_{t} \tilde{u}-\mu \partial_{x} \tilde{u}=a, & & \tilde{u}(\nu t, t)=u_{R}(t), \\
\partial_{t} \tilde{v}+\nu \partial_{x} \tilde{v}=c, & & \tilde{v}(-\mu t, t)=v_{L}(t) .
\end{aligned}
$$

Let $(x, t) \in D_{*}$. The (backward) characteristic $C_{-\mu}(x, t)$ crosses the (forward) characteristic $C_{\nu}(0)$ at time $T_{R}(x, t)=\frac{\mu t+x}{\mu+\nu}$. The characteristic $C_{\nu}(x, t)$ crosses the (forward) characteristic $C_{-\mu}(0)$ at time $T_{L}(x, t)=\frac{\nu t-x}{\mu+\nu}$. This provides

$$
\begin{aligned}
\tilde{u}(x, t) & =u_{R}\left(T_{R}(x, t)\right)+a\left(t-T_{R}(x, t)\right) \\
& =u_{R}^{0}+ \begin{cases}a t & \text { if } T_{R}(x, t)<\tau_{R}, \\
a \tau_{R}+b\left(T_{R}(x, t)-\tau_{R}\right)+a\left(t-T_{R}(x, t)\right) & \text { otherwise }\end{cases} \\
\tilde{v}(x, t) & =v_{L}\left(T_{L}(x, t)\right)+c\left(t-T_{L}(x, t)\right) \\
& =v_{L}^{0}+ \begin{cases}c t & \text { if } T_{L}(x, t)<\tau_{L}, \\
c \tau_{L}+d\left(T_{L}(x, t)-\tau_{L}\right)+c\left(t-T_{L}(x, t)\right) & \text { otherwise }\end{cases}
\end{aligned}
$$

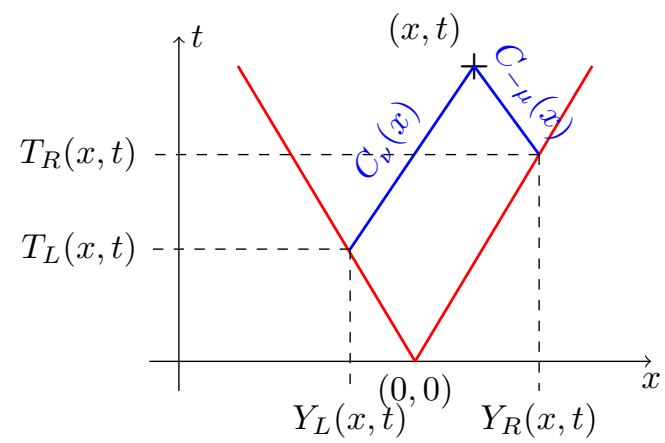

Fig. 15. Example of a configuration to compute $T_{L}(x, t)$ and $T_{R}(x, t)$ from a point $(x, t) \in D^{*}$. 
Computation of the boiling curve $\Gamma(\tilde{U})$ in $D^{*}$

We now prove that $\left\{(x, t) \in D_{*}: h(\tilde{U})(x, t)=0\right\}$ is overcharacteristic.

Lemma 6.3. Suppose that (6.1) holds. Then there exists a unique $\tau_{L}^{*}>0$ and a unique $\tau_{R}^{*}>0$ such that $h(\tilde{U})\left(-\mu \tau_{L}^{*}, \tau_{L}^{*}\right)=0$ and $h(\tilde{U})\left(\nu \tau_{R}^{*}, \tau_{R}^{*}\right)=0$. Moreover, there exists a unique function $T \in W^{1, \infty}(I)$ such that for all $(x, t) \in D^{*}$

$$
\left\{\begin{array}{lll}
h(\tilde{U})(x, t)<0 & \text { if and only if } & t<T(x), \\
h(\tilde{U})(x, t)=0 & \text { if and only if } & t=T(x), \\
h(\tilde{U})(x, t)>0 & \text { otherwise. } &
\end{array}\right.
$$

Furthermore, $T$ is continuous piecewise affine with at most three pieces and satisfies (3.1), i.e. its graph is overcharacteristic.

Proof. Decompose $D^{*}$ into (see Fig. 16)

$$
\begin{aligned}
& R_{1}=\left\{(x, t) \in D^{*}, \text { s.t. } T_{R}(x, t) \leq \tau_{R}, \text { and } T_{L}(x, t) \leq \tau_{L}\right\}, \\
& R_{2}=\left\{(x, t) \in D^{*}, \text { s.t. } T_{R}(x, t)>\tau_{R}, \text { and } T_{L}(x, t) \leq \tau_{L}\right\}, \\
& R_{3}=\left\{(x, t) \in D^{*}, \text { s.t. } T_{R}(x, t) \leq \tau_{R}, \text { and } T_{L}(x, t)>\tau_{L}\right\}, \\
& R_{4}=\left\{(x, t) \in D^{*} \text {, s.t. } T_{R}(x, t)>\tau_{R}, \text { and } T_{L}(x, t)>\tau_{L}\right\} .
\end{aligned}
$$

From the expressions (6.5) of $\tilde{u}$ and $\tilde{v}$ we get

$$
\begin{aligned}
\forall(x, t) \in R_{1}, & h(\tilde{U})(x, t)=h\left(u_{R}^{0}, v_{L}^{0}\right)+h(a, c) t \\
\forall(x, t) \in R_{2}, \quad h(\tilde{U})(x, t)= & h\left(u_{R}^{0}, v_{L}^{0}\right)+t h\left(\frac{a \nu+b \mu}{\mu+\nu}, c\right)+h_{1}(a-b)\left(\tau_{R}-\frac{x}{\mu+\nu}\right), \\
\forall(x, t) \in R_{3}, \quad h(\tilde{U})(x, t)= & h\left(u_{R}^{0}, v_{L}^{0}\right)+t h\left(a, \frac{d \nu+c \mu}{\mu+\nu}\right)+h_{2}(c-d)\left(\tau_{L}+\frac{x}{\mu+\nu}\right), \\
\forall(x, t) \in R_{4}, \quad h(\tilde{U})(x, t)= & h\left(u_{R}^{0}, v_{L}^{0}\right)+t h\left(\frac{a \nu+b \mu}{\mu+\nu}, \frac{d \nu+c \mu}{\mu+\nu}\right) \\
& +h\left((a-b)\left(\tau_{R}-\frac{x}{\mu+\nu}\right),(c-d)\left(\tau_{L}+\frac{x}{\mu+\nu}\right)\right) .
\end{aligned}
$$

As a consequence, $h(\tilde{U})(x, t)=0$ if and only if

$$
\begin{aligned}
& \text { in } R_{1}, \quad t=\frac{-h\left(u_{R}^{0}, v_{L}^{0}\right)}{h(a, c)}, \\
& \text { in } R_{2}, \quad t=\frac{-h\left(u_{R}^{0}+(a-b)\left(\tau_{R}-\frac{x}{\mu+\nu}\right), v_{L}^{0}\right)}{h\left(\frac{a \nu+b \mu}{\mu+\nu}, c\right)}, \\
& \text { in } R_{3}, \quad t=\frac{-h\left(u_{R}^{0}, v_{L}^{0}+(c-d)\left(\tau_{L}+\frac{x}{\mu+\nu}\right)\right)}{h\left(a, \frac{c \mu+d \nu}{\mu+\nu}\right)},
\end{aligned}
$$




$$
\text { in } R_{4}, \quad t=\frac{-h\left(u_{R}^{0}+(a-b)\left(\tau_{R}-\frac{x}{\mu+\nu}\right), v_{L}^{0}+(c-d)\left(\tau_{L}+\frac{x}{\mu+\nu}\right)\right)}{h\left(\frac{a \nu+b \mu}{\mu+\nu}, \frac{c \mu+d \nu}{\mu+\nu}\right)} \text {. }
$$

Remark that $\tilde{u}$ and $\tilde{v}$ are continuous in $D^{*}$, thus $h(\tilde{U})$ is continuous. Then (6.6) defines a function $T$ continuous piecewise affine. Using (6.1), one verifies that the $x$-derivative of (6.6) satisfies (4.5).

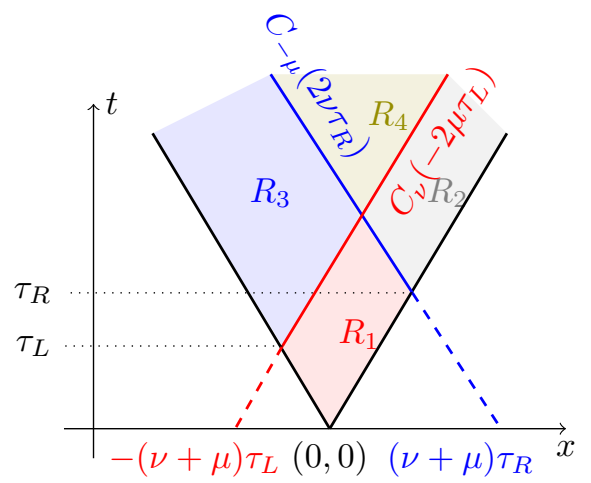

Fig. 16. Representation of the four regions of $D^{*}$ in which $\tilde{u}$ and $\tilde{v}$ are linear.

Correction of the solution for long time $\bar{U}$ in $D^{*}$

Now, we correct the short time solution $\tilde{U}$ so that the source term is $(b, d)$ above the boiling curve $\Gamma(\tilde{U})$ as it should be. Define

$$
\begin{aligned}
& \bar{u}(x, t)=u_{R}\left(T_{R}(x, t)\right)+a \mathcal{T}(x, t)+b\left(\left(t-T_{R}(x, t)\right)-\mathcal{T}(x, t)\right), \\
& \bar{v}(x, t)=v_{L}\left(T_{L}(x, t)\right)+c \mathfrak{T}(x, t)+d\left(\left(t-T_{L}(x, t)\right)-\mathfrak{T}(x, t)\right), \\
& \mathcal{T}(x, t)=\int_{T_{R}(x, t)}^{t} \mathbb{1}_{\mathbb{R}^{-}}(h(\tilde{u}, \tilde{v})(x+\mu(t-\tau), \tau)) d \tau, \\
& \mathfrak{T}(x, t)=\int_{T_{L}(x, t)}^{t} \mathbb{1}_{\mathbb{R}^{-}}(h(\tilde{u}, \tilde{x})(x-\nu(t-\tau), \tau)) d \tau .
\end{aligned}
$$

The characteristic $C_{-\mu}(x, t)$ in $D^{*}$ consists of two parts, one below the boiling line $\Gamma(\tilde{U})$ in the time interval $t \in\left[T_{R}(x, t), \mathcal{T}(x, t)\right]$ where the source term is $a$, the other above the boiling line in the time interval $t \in\left[T_{R}(x, t), \mathcal{T}(x, t)\right]$ and where the source is $b$. The two graphs intersect at most once since $T$ is overcharacteristic. Similarly, $\mathfrak{T}(x, t)$ plays the same role along the characteristic $C_{\nu}(x, t)$ (see Fig. 17). 
Explicit computations (Appendix Appendix C) give

$$
\begin{aligned}
& \mathcal{T}(x, t)= \begin{cases}0 & \text { if } \tau_{R}^{*} \leq T_{R}(x, t), \\
t-T_{R}(x, t) & \text { if } t \leq T(x), \\
\mathfrak{t}\left(T_{R}(x, t)\right)-T_{R}(x, t) & \text { otherwise, }\end{cases} \\
& \mathfrak{T}(x, t)= \begin{cases}0 & \text { if } \tau_{L}^{*} \leq T_{L}(x, t), \\
t-T_{L}(x, t) & \text { if } t \leq T(x), \\
\tau\left(T_{L}(x, t)\right)-T_{L}(x, t) & \text { otherwise, }\end{cases}
\end{aligned}
$$

where $\tau_{R}^{*}$ and $\tau_{L}^{*}$ are the times at which ends and starts $\Gamma^{*}$, i.e. the times spent in $Z^{-}(\tilde{U})$ along $C_{\nu}(0)$ and along $C_{-\mu}(0)$. It yields (see Appendix Appendix $\mathrm{C}$ )

$$
\tau_{R}^{*}=T\left((I d-\nu T)^{-1}(0)\right), \quad \tau_{L}^{*}=T\left((I d+\mu T)^{-1}(0)\right) .
$$

And $\mathfrak{t}(t)$, resp. $\tau(t)$, are the times spent in $Z^{-}(\tilde{U})$ along $C_{-\mu}(\nu t, t)$, resp. $C_{\nu}(-\mu t, t)$, i.e. starting at the point $(\nu t, t) \in C_{\nu}(0)$, resp. $(-\mu t, t) \in C_{-\mu}(0)$. It yields (see Appendix Appendix C)

$$
\mathfrak{t}(t)=T\left((I d+\mu T)^{-1}((\mu+\nu) t)\right), \quad \tau(t)=T\left((I d-\nu T)^{-1}(-(\mu+\nu) t)\right) .
$$

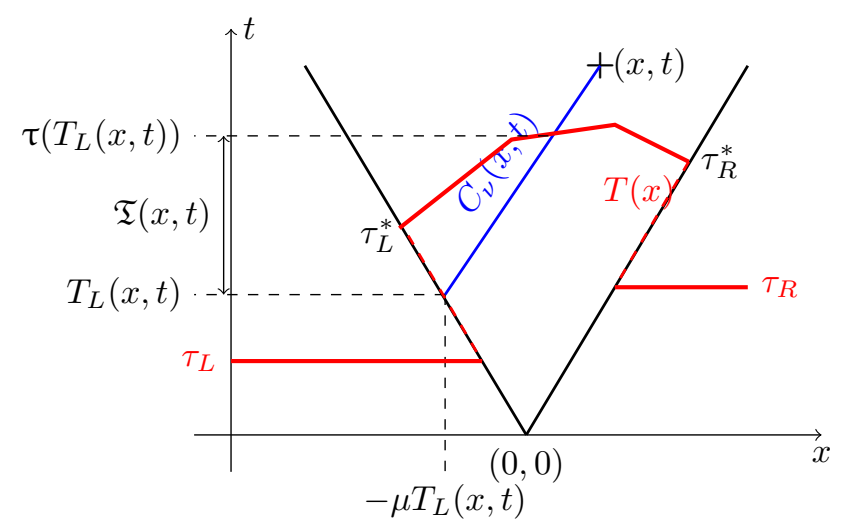

Fig. 17. Example of configuration for the computation of $\mathfrak{T}$.

\section{Conclusion}

We now verify that $\bar{U}$ is indeed a solution to the Riemann problem. The key point is that $h(\bar{U})$ is strictly increasing along the characteristics, thus is crosses the critical value 0 at most once. Before this time, the source term is $(a, c)$ and $\bar{U}=\tilde{U}$; after this time, the source term is $(b, d)$ as in the definition of $\bar{U}$. The proof is an adaptation of Section 4.2. 
Lemma 6.4. Suppose that (6.1) holds. Then, $\bar{U}=(\bar{u}, \bar{v})$ given by (6.7-6.8) satisfies

$$
\left(\partial_{t}-\mu \partial_{x}\right) h(\bar{U})>0, \quad\left(\partial_{t}+\nu \partial_{x}\right) h(\bar{U})>0 \quad \text { a.e. in }(x, t) \in D^{*} .
$$

Proof. The PDE given by (4.10-4.11) and (4.12) is modified into

$$
\left(\partial_{t}-\mu \partial_{x}\right) \bar{v}(x, t)=\left(\partial_{t}-\mu \partial_{x}\right) T_{L}(x, t)\left[v_{L}^{\prime}\left(T_{L}(x, t)\right)-d\right]+\left(\partial_{t}-\mu \partial_{x}\right) \mathfrak{T}(x, t)(c-d)+d,
$$

$\left(\partial_{t}+\nu \partial_{x}\right) \bar{u}(x, t)=\left(\partial_{t}+\nu \partial_{x}\right) T_{R}(x, t)\left[u_{R}^{\prime}\left(T_{R}(x, t)\right)-b\right]+\left(\partial_{t}+\nu \partial_{x}\right) \mathcal{T}(x, t)(a-b)+b$.

One verifies that

$$
\begin{aligned}
\left(\partial_{t}-\mu \partial_{x}\right) T_{R}(x, t) & =0, & & \left(\partial_{t}+\nu \partial_{x}\right) T_{R}(x, t)=1, \\
\left(\partial_{t}+\nu \partial_{x}\right) T_{L}(x, t) & =0, & & \left(\partial_{t}-\mu \partial_{x}\right) T_{L}(x, t)=1,
\end{aligned}
$$

and the other derivatives read

$$
\begin{aligned}
\left(\partial_{t}+\nu \partial_{x}\right) \mathcal{T}(x, t) & =\left\{\begin{array}{ll}
0 & \text { if } \tau_{R}^{*}<T_{R}(x, t), \\
0 & \text { if } t<T(x), \\
\mathfrak{t}^{\prime}\left(T_{R}(x, t)\right)-1
\end{array}\right. \text { otherwise, } \\
\left(\partial_{t}-\mu \partial_{x}\right) \mathfrak{T}(x, t) & = \begin{cases}0 & \text { if } \tau_{L}^{*}<T_{L}(x, t), \\
0 & \text { if } t<T(x), \\
\tau^{\prime}\left(T_{L}(x, t)\right)-1 & \text { otherwise, }\end{cases} \\
\mathfrak{t}^{\prime}(t) & =\frac{(\mu+\nu) T^{\prime}}{1+\mu T^{\prime}}\left((I d+\mu T)^{-1}((\mu+\nu) t)\right), \\
\tau^{\prime}(t) & =\frac{-(\mu+\nu) T^{\prime}}{1-\nu T^{\prime}}\left((I d-\nu T)^{-1}(-(\mu+\nu) t)\right) .
\end{aligned}
$$

Using the definition $h(x, T(x))=0$, one obtains

$$
T^{\prime}(x)=\frac{\partial_{x} h(\tilde{u}, \tilde{v})(x, T(x))}{\partial_{t} h(\tilde{u}, \tilde{v})(x, T(x))}=\frac{\left[\left(\partial_{t}-\mu \partial_{x}\right)-\left(\partial_{t}+\nu \partial_{x}\right)\right]}{\left[\nu\left(\partial_{t}-\mu \partial_{x}\right)+\mu\left(\partial_{t}+\nu \partial_{x}\right)\right]} h(\tilde{u}, \tilde{v})(x, T(x)),
$$

From (6.5), one computes

$$
\begin{aligned}
\left(\partial_{t}-\mu \partial_{x}\right) h(\tilde{U})(x, t) & =h\left(a, \varsigma_{L}(x, t)\right), & & \varsigma_{L}(x, t)=\varsigma\left(u_{L}, v_{L}\right)\left(T_{L}(x, t)\right) \\
\left(\partial_{t}+\nu \partial_{x}\right) h(\tilde{U})(x, t) & =h\left(\sigma_{R}(x, t), c\right), & & \sigma_{R}(x, t)=\sigma\left(u_{R}, v_{R}\right)\left(T_{R}(x, t)\right)
\end{aligned}
$$

which provides

$$
T^{\prime}(x)=\frac{h\left(a, \varsigma_{L}(x, T(x))\right)-h\left(\sigma_{R}(x, T(x)), c\right)}{\nu h\left(a, \varsigma_{L}(x, T(x))\right)+\mu h\left(\sigma_{R}(x, T(x)), c\right)},
$$

and leads to

$$
\begin{aligned}
\mathfrak{t}^{\prime}(t) & =1-\frac{h\left(\sigma_{R}\left(x_{R}(t), t_{R}(t)\right), c\right)}{h\left(a, \varsigma_{L}\left(x_{R}(t), t_{R}(t)\right)\right)}, & \tau^{\prime}(t) & =1-\frac{h\left(a, \varsigma_{L}\left(x_{L}(t), t_{L}(t)\right)\right)}{h\left(\sigma_{R}\left(x_{L}(t), t_{L}(t)\right), c\right)}, \\
x_{R}(t) & =(I d+\mu T)^{-1}((\mu+\nu) t), & x_{L}(t) & =(I d-\nu T)^{-1}(-(\mu+\nu) t), \\
t_{R}(t) & =T\left(x_{R}(t)\right), & t_{L}(t) & =T\left(x_{L}(t)\right) .
\end{aligned}
$$


Thus we get

$$
\begin{aligned}
\left(\partial_{t}-\mu \partial_{x}\right) h(\bar{U})(x, t) & =h\left(\sigma(\bar{U})(x, t), \varsigma_{L}(x, t)+(d-c) \alpha(x, t)\right), \\
\left(\partial_{t}+\nu \partial_{x}\right) h(\bar{U})(x, t) & =h\left(\sigma_{R}(x, t)+(b-a) \beta(x, t), \varsigma(\bar{U})(x, t)\right),
\end{aligned}
$$

with the coefficients

$$
\begin{aligned}
& \alpha(x, t)= \begin{cases}0 & \text { if } t<T(x) \quad \text { or } \quad \tau_{L}^{*}<T_{L}(x, t), \\
\frac{h\left(a, \varsigma_{L}(x, t)\right)}{h\left(\sigma_{R}(x, t), c\right)} & \text { otherwise, }\end{cases} \\
& \beta(x, t)= \begin{cases}0 & \text { if } t<T(x) \text { or } \tau_{R}^{*}<T_{R}(x, t), \\
\frac{h\left(\sigma_{R}(x, t), c\right)}{h\left(a, \varsigma_{L}(x, t)\right)} & \text { otherwise. }\end{cases}
\end{aligned}
$$

One concludes on the strict positivity from hypothesis (6.1):

- Either $t<T(x)$ or $\tau_{R}^{*}<T_{R}(x, t)$, resp. $\tau_{L}^{*}<T_{L}(x, t)$, then $\alpha=0$, resp. $\beta=0$, and (6.1a) provides the result.

- Or, $t>T(x)$ and $\Sigma(\bar{U})(x, t)=(b, d)$, and (6.10) turns into

$$
\begin{aligned}
\left(\partial_{t}-\mu \partial_{x}\right) h(\bar{U})(x, t) & =h\left(b, \varsigma_{L}(x, t)+(d-c) \frac{h\left(a, \varsigma_{L}(x, t)\right)}{h\left(\sigma_{R}(x, t), c\right)}\right) \\
& =\frac{h\left(a, \varsigma_{L}(x, t)\right)}{h\left(\sigma_{R}(x, t), c\right)}\left(\frac{h\left(\sigma_{R}(x, t), c\right)}{h\left(a, \varsigma_{L}(x, t)\right)} h\left(b, \varsigma_{L}(x, t)\right)+h_{2}(d-c)\right), \\
\left(\partial_{t}+\nu \partial_{x}\right) h(\bar{U})(x, t) & =h\left(\sigma_{R}(x, t)+(b-a) \frac{h\left(\sigma_{R}(x, t), c\right)}{h\left(a, \varsigma_{L}(x, t)\right)}, d\right) \\
& =\frac{h\left(\sigma_{R}(x, t), c\right)}{h\left(a, \varsigma_{L}(x, t)\right)}\left(\frac{h\left(a, \varsigma_{L}(x, t)\right)}{h\left(\sigma_{R}(x, t), c\right)} h\left(\sigma_{R}(x, t), d\right)+h_{1}(b-a)\right),
\end{aligned}
$$

which are strictly positive by hypothesis (6.1b) and (6.1c).

Finally, $\bar{U}$ is a generalized solution.

Lemma 6.5. Suppose that (6.1) holds. Then, (2.20) holds in $D^{*}$, and $U \in E$ defined by

$$
U(x, t)=U_{L}(x, t) \mathbb{1}_{D_{L}}(x, t)+\bar{U}(x, t) \mathbb{1}_{D^{*}}(x, t)+U_{R}(x, t) \mathbb{1}_{D_{R}}(x, t)
$$

is a generalized solution.

Proof. This is a straightforward adaptation of Lemma 4.6: $h(\bar{U})=h(\tilde{U})<0$ below $\Gamma^{*}$, then from the monotonicity of $h(\bar{U})$ along the characteristics and since $\Gamma^{*}$ is overcharacteristic, one obtains that $h(\bar{U})>0$ above $\Gamma^{*}$.

\subsection{Uniqueness of the generalized solution}

The uniqueness is again obtained by adapting the computations of Section 4, i.e. when (6.1) holds, then (6.7-6.8) is the unique generalized solution in $D^{*}$ and (6.11) is the unique generalized solution in $\mathbb{R} \times \mathbb{R}^{+}$. 


\subsection{Numerical illustration}

The solution $\bar{U}$ is illustrated through the numerical example

$$
\begin{aligned}
\partial_{t} u-\partial_{x} u & = \begin{cases}0.2 & \text { if } u+v \leq 0, \\
1 & \text { otherwise, }\end{cases} \\
\partial_{t} v+\partial_{x} v & = \begin{cases}1 & \text { if } u+v \leq 0, x) \\
2 & \text { otherwise, }\end{cases}
\end{aligned}
$$

over $(x, t) \in[0,4] \times[0,1]$. These data satisfy $(6.1)$.

This numerical solution is obtained using the upwind scheme

$$
\begin{aligned}
& u_{i}^{n+1}=u_{i}^{n}\left(1-\frac{\Delta t}{\Delta x}\right)+u_{i+1}^{n} \frac{\Delta t}{\Delta x}+ \begin{cases}0.2 \Delta t & \text { if } u_{i}^{n}+v_{i}^{n} \leq 0, \\
\Delta t & \text { otherwise },\end{cases} \\
& v_{i}^{n+1}=v_{i}^{n}\left(1-\frac{\Delta t}{\Delta x}\right)+v_{i-1}^{n} \frac{\Delta t}{\Delta x}+ \begin{cases}\Delta t & \text { if } u_{i}^{n}+v_{i}^{n} \leq 0, \\
2 \Delta t & \text { otherwise. }\end{cases}
\end{aligned}
$$

For general purposes, the use of an adapted discretization, especially of wellbalanced techniques such as upwinding the source $([4,15,22,16])$, is recommended to avoid numerical artifacts. The study of such adapted schemes for discontinuous sources is postponed to future work and (6.13) was found sufficient here and the qualitative results presented below are converged.

The spatial domain $x \in[0,4]$ is meshed with 800 cells and the time step is defined using a CFL of 0.95 . The numerical solution $(u, v)$ is plotted on Fig. 18 at the times $t=0.1, t=0.2, t=0.3, t=0.6$ and $t=1$. For each $t$, the solution $U=(u, v)$ is discontinuous at two points that move at velocity $-\mu$ and $\nu$. In $D_{L}$ and $D_{R}, U=U_{L}$ and $U=U_{R}$ is space independent, while it is piecewise linear in $D^{*}$. Though, the number of pieces in this center domain and their slopes vary in time.

This change in time is due to the switch of sign of $h(U)$. Where $h(U)$ changes sign, the derivatives of the solution $U$ become discontinuous. Then, the location of this change of regularity is transported along the characteristics. This phenomenon happens on the boundary of $D^{*}$ at the times $\tau_{L}$ and $\tau_{R}$ when $h\left(U_{L}\right)$ and $h\left(U_{R}\right)$ change sign, but also inside $D^{*}$. These lines where the solution loses its $C^{1}$ regularity are represented on Fig. 18 (bottom left) in green over the colormap of $h(U)$, and the yellow line is the line of discontinuity $\Gamma(U)$.

\section{Conclusion, discussions and outlooks}

This section gathers a summary of the results and a discussion about the constraints on the set of data $u_{0}, v_{0}, a, b, c$ and $d$.

\subsection{Conclusion}

In this paper we consider a $2 \times 2$ hyperbolic system with linear fluxes and a source term that is discontinuous in the unknown as a first attempt to study the numerical 

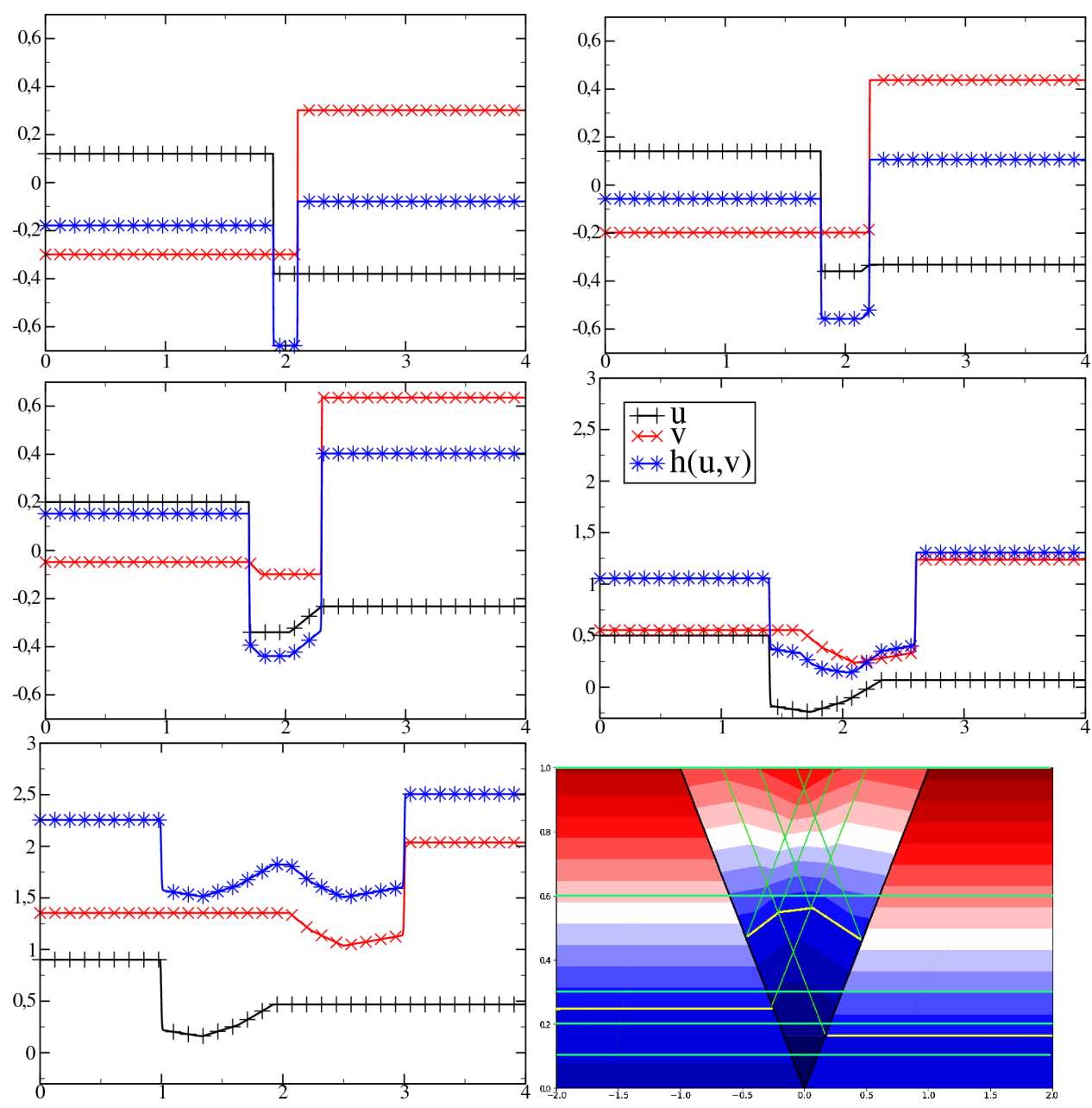

Fig. 18. Numerical solution $U=(u, v)$ to (6.12) at times $t=0.1$ (top left), $t=0.2$ (top right), $t=0.3$ (middle left), $t=0.6$ (middle right) and $t=1$ (bottom left), and representation (bottom right) of these times (in cyan) over the colormap of $h(u, v)$ in $(x, t)$-space.

modeling of flows with fast evolving sources, such as a boiling two-phase flow. Two important notions are introduced to study such problems. First, the solution is understood in an integral sense along the characteristics. Second, the source term jumps along a line in the $(x, t)$-space that is non-tangent (in a strong sense) to all the characteristic curves. These notions lead to prove the existence and uniqueness of a solution under different sets of restrictions over the data (initial conditions and value of the source) according to the regularity of the data: first in the case of regular initial conditions with either an overcharacteristic or a subcharacteristic line of discontinuity, then for Riemann problems.

Two relations between these sets of data are now discussed: 
- the relation between a Riemann problem $(1.2,3.5)$ in the limit $(\mu, \nu) \rightarrow(0,0)$ and a system of two ODEs with discontinuous RHS,

- the relation between the PDE (1.2) when the initial condition (satisfying (4.2)) tends to a Heaviside function and the resulting Riemann problem.

\subsection{The Riemann problem in the zero velocity limit}

Formally, when $(\mu, \nu) \rightarrow 0_{\mathbb{R}^{2}}$, the Riemann problem $(1.2,3.5)$ degenerates toward a system of two ODE's of the form (2.1), one for $x \in \mathbb{R}^{-}$and one for $x \in \mathbb{R}^{+}$. However, the requirement in Theorem 6.1 degenerates in a condition more restrictive than in Lemma 6.2 for the existence and uniqueness of a solution. Recall that (6.1) is sufficient but not necessary for the existence of a solution.

Indeed, one computes the solution $U$ from $(1.2,3.5)$ in the limit $(\mu, \nu) \rightarrow 0_{\mathbb{R}^{2}}$, and $U$ locally tends to

$$
u(x, t)=\left\{\begin{array}{ll}
u_{L}(t) & \text { if } \quad x \leq 0, \\
u_{R}(t) & \text { otherwise, }
\end{array} \quad v(x, t)= \begin{cases}v_{L}(t) & \text { if } \quad x \leq 0, \\
v_{R}(t) & \text { otherwise }\end{cases}\right.
$$

where $u_{L}, v_{L}, u_{R}$ and $v_{R}$ are defined in (6.3). By construction, these functions solve the ODE (6.4) and they exist under the condition $h(a, c) h(b, d)>0$.

For the existence of a solution to the Riemann problem, one remarks that the intensity of the velocities $\mu>0$ and $\nu>0$ have no influence on condition (6.1).

\subsection{The overcharacteristic problem in the limit of Heaviside initial condition}

The three theorems 3.3, 3.5 and 3.7 provide solutions under particular assumptions on the data $u_{0}, v_{0}, a, b, c, d$.

One remarks that the sets of data used in the different theorems are disjoint. For instance, the condition (4.2) on $u_{0}$ of Theorem 4.2 implies that inf $u_{0}^{\prime}>-\infty$ and $\sup v_{0}^{\prime}<\infty$. In particular, using this overcharacteristic framework, we may never consider the limit

$$
\left(u_{0}, v_{0}\right) \rightarrow\left(u_{L}, v_{L}\right)+\left(u_{R}-u_{L}, v_{R}-v_{L}\right) \mathbb{1}_{\mathbb{R}^{+}}
$$

with $u_{L}>u_{R}$ or $v_{L}<v_{R}$, corresponding to a Riemann problem. However, such a problem can be studied in the framework of Theorem 3.7 under condition (6.1).

This also implies that we may not expect the possibility of approximating such a problem with stiff initial data by a more regular one with the conditions that we have exhibited.

\subsection{Outlooks}

The main outlook to the present work consists in constructing numerical schemes for (1.1), possibly based on the method used to construct solutions to (1.2). A naive discretization of these equations may produce numerical artefacts on certain test 
cases, such as artificial oscillations, non-physical wave speeds, loss of admissibility (non-positive pressure or energy) or lack of preservation of equilibrium states. This impacts both the precision and the stability of the method. Using the scheme (6.13), we have been able to trigger such artefacts with a careful choice of the parameters $a, b, c, d, u_{0}$ and $v_{0}$. Note that the parameters (6.12) of Section 6.5 were also chosen carefully to avoid these. There is a large literature discussing the discretization of source terms in balance laws including the well-balanced schemes (see e.g. $[4,15,22$, $16]$ ), but it is mainly restricted to regular source terms. Extending these schemes to discontinuous source terms presents new difficulties, e.g. to obtain the existence of discrete steady states for (1.1). The present construction provides a solution to (1.2) and its behaviour in certain regimes. One may exploit the construction of Section 4 and 5 to construct well-balanced finite difference schemes or the one of Section 6 to construct well-balanced Godunov schemes for PDE with discontinuous sources.

Other extensions of the present work toward the general PDE (1.1) may be considered. The generalization from (1.2) to a system with $N$ equations with linear fluxes and non-linear enthalpy is expected to be only technical and to present no real difficulty. However, the extension to non-linear fluxes is much more complicated. Indeed, only contact discontinuities were considered here, and the present method may misbehave when shock and rarefaction waves are forming. Especially, the notion of integral solutions along the characteristics can be generalized with non-linear fluxes when the solution is smooth, but such a construction needs further work in the presence of shocks.

\section{Acknowledgement}

This work was funded by CEA and LJLL (Sorbonne University and CNRS) through LRC MANON.

\section{Appendix A. Computation of $T_{L}$ and $T_{R}$ in the Riemann problem}

Consider $(x, t) \in D^{*}$ and define $\left(T_{R}(x, t), Y_{R}(x, t)\right)$, resp. $\left(T_{L}(x, t), Y_{L}(x, t)\right)$, the point of intersection between $C_{-\mu}(x, t)$ and $C_{\nu}(0)$, resp. $C_{\nu}(x, t)$ and $C_{-\mu}(0)$. By definition, these points satisfy

$$
\begin{aligned}
& \left\{\begin{array} { l } 
{ Y _ { R } + \mu T _ { R } = x + \mu t , } \\
{ Y _ { R } - \nu T _ { R } = 0 , }
\end{array} \Rightarrow \left\{\begin{array}{l}
T_{R}(x, t)=\frac{\mu t+x}{\mu+\nu}, \\
Y_{R}(x, t)=\frac{\nu(\mu t+x)}{\mu+\nu},
\end{array}\right.\right. \\
& \left\{\begin{array} { l } 
{ Y _ { L } + \mu T _ { L } = 0 , } \\
{ Y _ { L } - \nu T _ { L } = x - \nu t , }
\end{array} \Rightarrow \left\{\begin{array}{l}
T_{L}(x, t)=\frac{\nu t-x}{\mu+\nu}, \\
Y_{L}(x, t)=\frac{-\mu(\nu t-x)}{\mu+\nu} .
\end{array}\right.\right.
\end{aligned}
$$




\section{Appendix B. Computation of $\tilde{\Sigma}$ in the Riemann problem}

Any generalized solution $U=(u, v) \in E$, especially $u$ remains continuous along $C_{-\mu}(x)$ and $v$ along $C_{\nu}(x)$ for all $x \neq 0$. The value of the sources $\tilde{\Sigma}$ in $D^{*}$ is chosen constant based on the following property.

Lemma Appendix B.1. Any generalized solution $U=(u, v)$ satisfies

$$
\lim _{\substack{(x, t) \rightarrow(0,0) \\(x, t) \in D^{*}}} h(U)(x, t)=h\left(u_{R}^{0}, v_{L}^{0}\right) .
$$

Proof. Using the method of characteristics leads to define the solution as

$$
\begin{aligned}
& u(x, t)=u_{R}\left(T_{R}(x, t)\right)+\int_{T_{R}(x, t)}^{t} \sigma(u, v)(x+\mu(t-\tau), \tau) d \tau, \\
& v(x, t)=v_{L}\left(T_{L}(x, t)\right)+\int_{T_{L}(x, t)}^{t} \varsigma(u, v)(x-\nu(t-\tau), \tau) d \tau,
\end{aligned}
$$

where $u_{R}$ and $v_{L}$ are given by (6.3). One computes

$$
\begin{aligned}
h(U)(x, t) & =h\left(u_{R}\left(T_{R}(x, t)\right), v_{L}\left(T_{L}(x, t)\right)\right) \\
& +h\left(\int_{T_{R}(x, t)}^{t} \sigma(u, v)(x+\mu(t-\tau), \tau) d \tau, \int_{T_{L}(x, t)}^{t} \varsigma(u, v)(x-\nu(t-\tau), \tau) d \tau\right) .
\end{aligned}
$$

Since $\sigma$ is bounded by $a$ and $b$ and $\varsigma$ by $c$ and $d$, the integrals in this formula are bounded. By continuity of $T_{R}, T_{L}$ and $h$, computing $h(U)$ in the limit $D^{*} \ni(x, t) \rightarrow$ $(0,0)$ provides the result.

\section{Appendix C. Details on the computation of $\mathcal{T}$ and $\mathfrak{T}$ in $D^{*}$}

Since $\Gamma^{*}$ is overcharacteristic, for all $(x, t) \in D^{*}$, the characteristic curves $C_{-\mu}(x, t)$ and $C_{\nu}(x, t)$ cross $\Gamma^{*}$ at most once.

- Either $t<T(x)$ then the part of the characteristics in $D^{*}$ and before time $t$ (see Fig. 17) are below $\Gamma^{*}$ and thus entirely in $Z^{-}(U)$. Then the term in the integral (6.7) is constant equal to one and

$$
\mathcal{T}(x, t)=t-T_{R}(x, t) \quad \text { if } \quad t \leq T(x), \quad \mathfrak{T}(x, t)=t-T_{L}(x, t) \quad \text { if } \quad t \leq T(x) .
$$

- Denote $\tau_{R}^{*}$ and $\tau_{L}^{*}$ the points such that

$$
\left(-\mu \tau_{L}^{*}, \tau_{L}^{*}\right) \in C_{-\mu}(0) \cap \Gamma^{*}, \quad\left(\nu \tau_{R}^{*}, \tau_{R}^{*}\right) \in C_{\nu}(0) \cap \Gamma^{*},
$$

i.e. the starting and ending points of $\Gamma^{*}$ in $D^{*}$.

Then, consider a point $(x, t) \in D^{*}$ such that $T_{R}(x, t) \geq \tau_{R}^{*}$ and $T_{L}(x, t) \geq \tau_{L}^{*}$. Again since $\Gamma^{*}$ is overcharacteristic, the part of the characteristics $C_{-\mu}(x, t)$ and $C_{\nu}(x, t)$ in $D^{*}$ and before time $t$ are entirely in $Z^{+}(\tilde{U})$. Then the term in the integral (6.7) is constant equal to zero and

$$
\mathcal{T}(x, t)=0 \quad \text { if } \quad T_{R}(x, t) \geq \tau_{R}^{*}, \quad \mathfrak{T}(x, t)=0 \quad \text { if } \quad T_{L}(x, t) \geq \tau_{L}^{*} .
$$


The values of $\tau_{L}^{*}$ and $\tau_{R}^{*}$ satisfy

$$
\begin{aligned}
& \left\{\begin{array} { r l } 
{ Y _ { L } ^ { * } + \mu \tau _ { L } ^ { * } } & { = 0 , } \\
{ T ( Y _ { L } ^ { * } ) } & { = \tau _ { L } ^ { * } , }
\end{array} \Rightarrow \left\{\begin{array}{r}
Y_{L}^{*}=(I d+\mu T)^{-1}(0), \\
\tau_{L}^{*}=T\left((I d+\mu T)^{-1}(0)\right),
\end{array}\right.\right. \\
& \left\{\begin{array} { r } 
{ Y _ { R } ^ { * } - \nu \tau _ { R } ^ { * } = 0 , } \\
{ T ( Y _ { R } ^ { * } ) = \tau _ { R } ^ { * } , }
\end{array} \Rightarrow \left\{\begin{array}{r}
Y_{R}^{*}=(I d-\nu T)^{-1}(0), \\
\tau_{R}^{*}=T\left((I d-\nu T)^{-1}(0)\right) .
\end{array}\right.\right.
\end{aligned}
$$

These values are obtained along $C_{-\mu}\left(0^{+}\right)$and $C_{\nu}\left(0^{-}\right)$when $h(\tilde{U})$ reaches the value 0 . Along these lines, one computes

$$
\begin{aligned}
\forall(x, t) \in C_{-\mu}\left(0^{+}\right) \cap Z^{-}(\tilde{U}), \quad h(\tilde{U})(x, t) & =h\left(u_{R}^{0}+a t, v_{L}(t)\right) \\
& =\left\{\begin{array}{l}
h\left(u_{R}^{0}, v_{L}^{0}\right)+h(a, c) t \quad \text { if } t \leq \tau_{L}, \\
h\left(u_{R}^{0}, v_{L}^{0}+(c-d) \tau_{L}\right)+h(a, d) t \quad \text { otherwise, }
\end{array}\right. \\
\forall(x, t) \in C_{\nu}\left(0^{-}\right) \cap Z^{-}(\tilde{U}), \quad h(\tilde{U})(x, t) & =h\left(u_{R}(t), v_{L}^{0}+c t\right) \\
& =\left\{\begin{array}{l}
h\left(u_{R}^{0}, v_{L}^{0}\right)+h(a, c) t \text { if } t \leq \tau_{R}, \\
h\left(u_{R}^{0}+(a-b) \tau_{R}, v_{L}^{0}\right)+h(b, c) t \quad \text { otherwise, }
\end{array}\right.
\end{aligned}
$$

which provides

$$
\begin{array}{r}
h(\tilde{U})(-\mu t, t)=0 \quad \Leftrightarrow \quad t=\tau_{L}^{*}=\left\{\begin{array}{l}
\frac{-h\left(u_{R}^{0}, v_{L}^{0}\right)}{h(a, c)} \text { if } \frac{-h\left(u_{R}^{0}, v_{L}^{0}\right)}{h(a, c)}<\tau_{L}, \\
\frac{-h\left(u_{R}^{0}, v_{L}^{0}+(c-d) \tau_{L}\right)}{h(a, d)} \text { otherwise, }
\end{array}\right. \\
h(\tilde{U})(\nu t, t)=0 \quad \Leftrightarrow \quad t=\tau_{R}^{*}= \begin{cases}\frac{-h\left(u_{R}^{0}, v_{L}^{0}\right)}{h(a, c)} \text { if } \frac{-h\left(u_{R}^{0}, v_{L}^{0}\right)}{h(a, c)}<\tau_{R}, \\
\frac{-h\left(u_{R}^{0}+(a-b) \tau_{R}, v_{L}^{0}\right)}{h(b, c)} & \text { otherwise. }\end{cases}
\end{array}
$$

- Otherwise, at $(x, t) \in D^{*}$, the characteristic curve $C_{-\mu}(x, t)$, or $C_{\nu}(x, t)$, has crossed once $\Gamma^{*}$. Then the term in the integral (6.7) is one before $\mathfrak{t}$, resp. $\tau$, and 0 after, then these integrals rewrite

$$
\begin{array}{lllll}
\mathcal{T}(x, t)=\mathfrak{t}\left(T_{R}(x, t)\right)-T_{R}(x, t) & \text { if } & t<T(x) & \text { and } & T_{R}(x, t) \leq \tau_{R}^{*}, \\
\mathfrak{T}(x, t)=\tau\left(T_{L}(x, t)\right)-T_{L}(x, t) & \text { if } \quad t<T(x) & \text { and } & T_{L}(x, t) \leq \tau_{L}^{*} .
\end{array}
$$

Here, $\mathfrak{t}\left(T_{R}(x, t)\right)$, resp. $\tau\left(T_{L}(x, t)\right)$, is the time spent in $Z^{-}(\tilde{U})$ along the characteristic $C_{-\mu}(x, t)=C_{-\mu}\left(\nu T_{R}(x, t), T_{R}(x, t)\right)$, resp. $C_{\nu}(x, t)=$ $C_{\nu}\left(-\mu T_{L}(x, t), T_{L}(x, t)\right)$. Especially, these times satisfy

$$
(\mathfrak{x}, \mathfrak{t})(t) \in C_{-\mu}(\nu t, t) \cap \Gamma^{*}, \quad(\xi, \tau)(t) \in C_{\nu}(-\mu t, t) \cap \Gamma^{*},
$$

that rewrites

$$
\begin{gathered}
\left\{\begin{array}{c}
\mathfrak{x}(t)+\mu \mathfrak{t}(t)=(\nu+\mu) t, \\
T(\mathfrak{x}(t))=\mathfrak{t}(t),
\end{array}\right. \\
\left\{\begin{array} { c } 
{ \xi ( t ) - \nu \tau ( t ) = - ( \nu + \mu ) t , } \\
{ T ( \xi ( t ) ) = \tau ( t ) , }
\end{array} \Leftrightarrow \left\{\begin{array}{l}
\mathfrak{x}(t)=(I d+\mu T)^{-1}((\nu+\mu) t), \\
\mathfrak{t}(t)=T\left((I d+\mu T)^{-1}((\nu+\mu) t)\right),
\end{array}\right.\right. \\
\hline\left\{\begin{array}{l}
\xi(t)=(I d-\nu T)^{-1}(-(\nu+\mu) t), \\
\tau(t)=T\left((I d-\nu T)^{-1}(-(\nu+\mu) t)\right),
\end{array}\right.
\end{gathered}
$$

As for $\tau_{L}^{*}$ and $\tau_{R}^{*}, \mathfrak{t}$ and $\tau$ can be rewritten based on $u_{R}$ and $v_{L}$ and $a, b, c$ and $d$. 


\section{References}

[1] G. Allaire, X. Blanc, B. Desprs, and F. Golse. Transport et diffusion. Edition de l'cole polytechnique, 2019

[2] J.-P. Aubin and A. Cellina. Differential Inclusions, Set-Valued Maps And Viability Theory. Grundl. der Math. Wiss. Springer, 1984.

[3] A. Bergeron and I. Toumi. Assessment of the FLICA-IV code on rod bundle experiments. Proceedings of ICONE-6, San Diego, California, USA, 1998.

[4] A. Bermudez and M. E. Vazquez. Upwind methods for hyperbolic conservation laws with source terms. Comput. \& Fluids, 23(8):1049-1071, 1994.

[5] A. Bressan. Unique solutions for a class of discontinuous differential equations. Proceedings of the American Mathematical Society, 104(3):772-778, 1988.

[6] A. Bressan. Chapter 3. In Hyperbolic systems of conservation laws: The onedimensional Cauchy problem. Oxford University Press, 2000.

[7] A. Bressan and G. Colombo. Existence and continuous dependence for discontinuous O.D.E.'s. Boll. Un. Mat. Ital. 4-B, pages 295-311, 1909.

[8] H. Brézis. Operateurs maximaux monotones et semi-groupes de contractions dans les espaces de Hilbert. North-Holland publishing company, 1973.

[9] H. B. Callen. Thermodynamics and an introduction to thermostatics. John Wiley \& Sons, 1985.

[10] D. Campbell, S. Hencl, and F. Konopeck. The weak inverse mapping theorem. $Z$. Anal. Anwendungen, 34:321-342, 2015.

[11] C. Carathéodory. Paragraphs 13-20. In Calculus of variations and partial differential equations of the first order, pages 13-23. AMS Chelsea publishing, 1999.

[12] C. L. Evans. Section 3.2. In Partial differential equations, pages 97-115. American Mathematical Society, 2010.

[13] A. F. Filippov. Differential Equations with Discontinuous Righthand Sides. Springer, 1988.

[14] E. Godlewski and P.-A. Raviart. Section 1.5. In Numerical Approximation of Hyperbolic Systems of Conservation Laws, pages 70-82. Springer, 1996.

[15] L. Gosse. Section 4.4. In Computing Qualitatively Correct Approximations of Balance Laws, pages 70-76. Springer, 2013.

[16] J. M. Greenberg and A. Y. Leroux. A well-balanced scheme for the numerical processing of source terms in hyperbolic equations. SIAM J. Numer. Anal., 33(1):116, 1994.

[17] E. Hairer and G. Wanner. Solving Ordinary Differential Equations II. Stiff and Differential-Algebraic Problems. Springer Verlag Berlin, 1996.

[18] O. Hajek. Discontinuous differential equations. Int. J. Differential Equations, 32:149$170,1979$.

[19] T. Hibiki and M. Ishii. One-dimensional drift-flux model and constitutive equations for relative motion between phases in various two-phase flow regimes. Int. J. Heat Mass Transfer, 46:4935-4948, 2003.

[20] M. Ishii. One dimensional drift-flux model and constitutive equations for relative motion between phases in various two-phase flow. Technical report, ANL, 1977.

[21] M. Ishii and T. Hibiki. Thermo-fluid dynamics of two-phase flows. Springer, 2011.

[22] R. J. Leveque and H. C. Yee. A study of numerical methods for hyperbolic conservation laws with stiff source terms. Technical report, NASA, 1988.

[23] C. Lobry and T. Sari. quations diffrentielles second membre discontinu. Contrle non linaire et Applications, (64):255-289, 2005.

[24] T. Pichard. Existence of steady two-phase flows with discontinuous boiling effects. AIMS, Proceedings of HYP18 conference, accepted. 
[25] A. Pucci. Sistemi di equazioni differenziali con secondo membro discontinuo rispetto all'incognita. Rend. Ist. Mat. Univ. Trieste, III:75-80, 1971.

[26] E. Royer, S. Aniel, A. Bergeron, P. Fillion, D. Gallo, F. Gaudier, O. Grégoire, M. Martin, E. Richebois, P. Salvadore, S. Zimmer, T. Chataing, P. Clément, and F. François. FLICA4: status of numerical and physical models and overview of applications. Proceedings of NURETH-11, Avignon, France, 2005.

[27] I. Toumi, A. Bergeron, D. Gallo, E. Royer, and D. Caruge. FLICA-4: a threedimensional two-phase flow computer code with advanced numerical methods for nuclear applications. Nuclear Engineering and Design, 200, 2000.

[28] G. B. Whitam. Section 5.1-2. In Linear and nonlinear waves, pages 114-123. John Wiley \& sons, inc., 1999. 